\title{
BOUNDARY BEHAVIOR OF SOLUTIONS TO THE PARABOLIC $p$-LAPLACE EQUATION
}

\author{
BENNY AVELIN, TUOMO KUUSI, AND KAJ NYSTRÖM
}

\begin{abstract}
We establish boundary estimates for non-negative solutions to the $p$-parabolic equation in the degenerate range $p>2$. Our main results include new parabolic intrinsic Harnack chains in cylindrical NTA-domains together with sharp boundary decay estimates. If the underlying domain is $C^{1,1}$-regular, we establish a relatively complete theory of the boundary behavior, including boundary Harnack principles and Hölder continuity of the ratios of two solutions, as well as fine properties of associated boundary measures. There is an intrinsic waiting time phenomena present which plays a fundamental role throughout the paper. In particular, conditions on these waiting times rule out well-known examples of explicit solutions violating the boundary Harnack principle.
\end{abstract}

\section{INTRODUCTION AND RESULTS}

This paper is devoted to a study of the boundary behavior of non-negative solutions to the $p$-parabolic equation, in the degenerate range $p>2$. We restrict the analysis to space-time cylinders $\Omega_{T}=\Omega \times(0, T), T>0$, where $\Omega \subset \mathbb{R}^{n}$ is a bounded domain, i.e., an open and connected set. Given $p, 1<p<\infty$, fixed, recall that the $p$-parabolic equation is the equation

$$
\partial_{t} u-\Delta_{p} u:=\partial_{t} u-\nabla \cdot\left(|\nabla u|^{p-2} \nabla u\right)=0 .
$$

In the special case $p=2$ the $p$-parabolic equation coincides with the heat equation, and in this case we refer to Kemper [19], Salsa [31], and also [13, 14, 15, $16,17,29]$, concerning the boundary behavior of non-negative solutions. Key results established in these works, in the context of Lipschitz-cylinders $\Omega_{T}$, include Carleson type estimates, the relation between the associate parabolic measure and Green function, the backward in time Harnack inequality, boundary Harnack principles (local and global) and Hölder continuity up to the boundary of quotients of non-negative solutions vanishing on the lateral boundary.

On the contrary for $p \neq 2,1<p<\infty$, much less is known concerning these problems and we refer the reader to [2, 3, 24] for accounts of the current literature. For a relatively complete picture in the case of non-linear parabolic operators with linear growth we refer to [30]. However, it is also important to mention that there is an interesting and related recent literature devoted to the asymptotic and pointwise behavior of solutions to non-linear diffusion equations on bounded domains, see [32], and also [8] for the porous medium type equations, and the references therein.

Considering non-negative solutions to the $p$-parabolic equation, for $p$ in the degenerate range $p>2$, it is a priori not clear to what extent and in what sense the above mentioned results can remain to hold. Indeed, on the one hand we have to account for the lack of homogeneity of the $p$-parabolic equation, and on the other

Date: July 18, 2018.

2010 Mathematics Subject Classification. 35K20, 35K65, 35B65.

Key words and phrases. p-parabolic equation, degenerate, intrinsic geometry, waiting time phenomenon, intrinsic Harnack chains, boundary Harnack principle, $p$-stability . 
hand we have to account for the fact that in the degenerate regime the phenomenon of finite speed propagation is present. As a matter of fact, simple examples show that in this case there are, compared to the case $p=2$, much more delicate waiting time phenomena to take into account.

To discuss the aspects of the waiting time phenomena further, we here first briefly describe some by now classical results in the case $p=2$, see $[14,31]$. Assume that $\Omega$ is, say, a Lipschitz domain, that $x_{0} \in \partial \Omega$ and let $A_{ \pm}:=\left(a_{r}\left(x_{0}\right), t_{0} \pm r^{2}\right)$, where $a_{r}\left(x_{0}\right)$ is an interior point of $\Omega$ with distance to the boundary comparable to $r$. Assume also that $u$ and $v$ are non-negative caloric functions in $\Omega_{T}$, i.e., functions satisfying (1.1) with $p=2$ in $\Omega_{T}$, vanishing continuously on $\left(\partial \Omega \cap B_{r}\left(x_{0}\right)\right) \times\left(t_{0}-r^{2}, t_{0}+r^{2}\right)$, where $B_{r}\left(x_{0}\right) \subset \mathbb{R}^{n}$ is the standard Euclidean ball of radius $r$ and centered at $x_{0} \in \mathbb{R}^{n}$. Then

$$
c^{-1} \frac{u\left(A_{-}\right)}{v\left(A_{+}\right)} \leq \frac{u(x, t)}{v(x, t)} \leq c \frac{u\left(A_{+}\right)}{v\left(A_{-}\right)},
$$

for a universal constant $c$, whenever $(x, t) \in\left(\Omega \cap B_{r / 2}\left(x_{0}\right)\right) \times\left(t_{0}-(r / 2)^{2}, t_{0}+(r / 2)^{2}\right)$. However, in general an estimate like (1.2) dramatically fails in the case $p \neq 2$. To see this, recall the following two classical solutions (see e.g. [3]) in the case when $\Omega:=\mathbb{R}^{n-1} \times\left\{x_{n}: x_{n}>0\right\}:$

$$
u(x, t)=c_{p}(T-t)^{-1 /(p-2)} x_{n}^{p /(p-2)}, \quad v(x, t)=x_{n} .
$$

In view of the examples in (1.3) it is not clear under what conditions the boundary Harnack principle in (1.2) could hold. Let us make a few observations. When defining $u$ as in (1.3), we see that the larger we take $T$, the longer the solution $u$ exists and the smaller its pointwise values become at a fixed time $t<T$. If we wish to show an estimate as in (1.2), we need to be able to rule out examples like $u$ in (1.3) (see also some other examples in [3]). We do this by simply requiring, for $(x, t) \in \Omega_{T}$ fixed, that

$$
T-t>C_{0} u(x, t)^{2-p} d(x, \partial \Omega)^{p},
$$

for a large enough constant $C_{0}$. It is easily seen that the solution $u$ in (1.3) does not satisfy (1.4) at any point $(x, t) \in \Omega_{T}$ if we require $C_{0} \geq c_{p}^{p-2}$.

In this context, and for the $p$-parabolic equation, it is here natural to make a link to the by now classical method of intrinsic scaling due to DiBenedetto, see [9]. The intrinsic scalings define the canonical geometry in which weak solutions to the $p$-parabolic equation become homogenized in a sense to be made precise. Indeed, in this geometry we consider, instead of the standard parabolic cylinders, intrinsically time-scaled cylinders of the type

$$
\begin{aligned}
& Q_{r}^{\lambda,+}(x, t):=B_{r}(x) \times\left(t, t+\lambda^{2-p} r^{p}\right), \\
& Q_{r}^{\lambda,-}(x, t):=B_{r}(x) \times\left(t-\lambda^{2-p} r^{p}, t\right), \quad \lambda:=u(x, t) .
\end{aligned}
$$

These kinds of intrinsic cylinders appear naturally in the context of Harnack inequalities, oscillation reduction estimates, and decay estimates, and defines the correct geometry in our setting.

The main goal of this paper is to study to what extent the theory developed in [14, 31] generalizes to the case $p>2$, under suitable intrinsic conditions. We have already seen that it rules out the pathological examples like $u$ in (1.3). In fact, we prove that (1.4) is a sufficient condition for developing a rather general theory concerning the boundary behavior of non-negative solutions to the $p$-parabolic equation. For instance, (1.4) allows us to prove a counterpart of (1.2) valid for $2<p<\infty$, see Theorem 9.4 below.

1.1. Summary of results. We will now give an informal summary of our results. The precise statements can be found in the bulk of the paper. 
Harnack chains. Fundamental tools in the study of the boundary behavior of nonnegative solutions are the Harnack inequality and Harnack chains. Harnack chains allow one to relate the value of non-negative solutions at different space-time points in the domain. For $p=2$ the Harnack inequality is homogeneous and, roughly, to control the values of the solution in a ball of size $r$ requires a waiting time comparable to $r^{2}$. For $p>2$ we have to use an intrinsic version of the Harnack inequality $[9,11]$. In particular the intrinsic Harnack inequality states, see Theorem 3.1, that if we have a non-negative solution $u$ to the $p$-parabolic equation in $\Omega_{T}$, with $(x, t) \in \Omega_{T}$, and $Q_{4 r}^{u(x, t) / c_{h},-}(x, t) \subset \Omega_{T}$, then

$$
u(x, t) \leq C_{h} \inf _{y \in B_{r}(x)} u\left(y, t+\left[\frac{c_{h}}{u(x, t)}\right]^{p-2} r^{p}\right)
$$

provided that $t+\left[\frac{c_{h}}{u(x, t)}\right]^{p-2} r^{p}<T$. The intrinsic waiting time required in this Harnack inequality is consistent with the condition stated in (1.4). In Section 3 we develop a sequence of Harnack chain estimates and the goal of that section is twofold. First, we want to establish estimates applicable in cylindrical NTAdomains, see Definition 2.1. Second, we want to establish a $p$-stable counterpart of the sharp Harnack chain estimate proved by Salsa [31, Theorem C], which in the case $p=2$ reads as

$$
u(y, s) \leq u(x, t) \exp \left[C\left(\frac{|x-y|^{2}}{t-s}+\frac{t-s}{k}+1\right)\right],
$$

where $k=\min \left\{1, s, d(x, \partial \Omega)^{2}, d(y, \partial \Omega)^{2}\right\}, s<t$, and $(x, t),(y, s) \in \Omega_{T}$. To do this we develop Harnack chains based on the weak Harnack inequality proved in [22], see Theorem 3.2 below, valid for supersolutions to the $p$-parabolic equation. As truncations of our solutions are supersolutions to (1.1), we are able to control the waiting times more precisely by adjusting the levels at which the solutions are truncated. This is in sharp contrast to the Harnack chains developed in [2, 3] for which there is very little control over the waiting time. Our approach to Harnack chains has at least three advantages. First, it allows us to construct Harnack chains starting from the measure $u\left(x, t_{0}\right) \mathrm{d} x$ at the initial time $t_{0}$. Second, it allows us to develop a flexible Carleson estimate, see Section 4, generalizing the one in [3] and which, in addition, remains valid in the context of time-independent NTA-cylinders. We note that although the Carleson estimate proved in [2] is valid in the setting of time-independent NTA-cylinders, a difference compared to the results in this paper is that the Carleson estimate proved in [2] is not $p$-stable as $p \rightarrow 2$. Third, we develop a version of (1.5) which is $p$-stable in the sense that we recover (1.5) as $p \rightarrow 2$. To establish this Gaussian type behavior for $p>2$ is technically rather involved.

Estimates of associated boundary measures. In the study of the boundary behavior of quasi-linear equations of $p$-Laplace type, certain Riesz measures supported on the boundary and associated to non-negative solutions vanishing on a portion of the boundary are important, see [26, 27]. These measures are non-linear generalizations of the harmonic measure relevant in the study of the Laplace equation and the Green function. Corresponding measures can also be associated to solutions to the $p$-parabolic equation. Indeed, let $u$ be a non-negative solution in $\Omega_{T}$, assume that $u$ is continuous on the closure of $\Omega_{T}$, and that $u$ vanishes on $\partial_{p} \Omega_{T} \cap Q$ with some open set $Q$. Extending $u$ to be zero in $Q \backslash \Omega_{T}$, it is straightforward to see that $u$ is a continuous weak subsolution to (1.1) in $Q$. Using this, one can conclude that there exists a unique locally finite positive Borel measure $\mu$, supported on $S_{T} \cap Q$, 
such that

$$
\int_{Q} u \partial_{t} \phi \mathrm{d} x \mathrm{~d} t-\int_{Q}|\nabla u|^{p-2} \nabla u \cdot \nabla \phi \mathrm{d} x \mathrm{~d} t=\int_{Q} \phi \mathrm{d} \mu
$$

whenever $\phi \in C_{0}^{\infty}(Q)$. In Section 5 we establish, in cylindrical NTA-domains, both upper and lower bounds for the measure $\mu$ in terms of $u$. If $\Omega$ is smooth, then $\mathrm{d} \mu=|\nabla u|^{p-1} \mathrm{~d} H^{n-1} \mathrm{~d} t$. Based on this the lower bound established on $\mu$ can be interpreted as a non-degeneracy estimate, close to the boundary, of the solution. Our proof of the lower bound for the measure $\mu$ is a modification of the elliptic proof, see for example [5, 21]. However, our proof is genuinely non-linear, it applies to much more general operators of $p$-parabolic type, and the result seems to be new already in the case $p=2$.

$A$ "complete theory" in $C^{1,1}$-domains. We establish a "complete theory" concerning the boundary behavior of non-negative solutions in $\Omega_{T}$ in the case when $\Omega$ is a $C^{1,1_{-}}$ domain. As a comprehensive literature is missing, we in Sections 8 and 9 develop both a local as well as a global theory of boundary behavior in $C^{1,1}$-cylinders. In the global setting we are able, as in [14] with corresponding estimates in the case $p=2$, to give a rather complete picture. For nonnegative solutions vanishing on the lateral boundary, our results include a global boundary Harnack principle and Hölder continuity of ratios. On the other hand, in the local setting we prove a new intrinsic local boundary Harnack principle. In the context of $C^{1,1}$-cylinders we are also able to show that the boundary measure in (1.6) is mutually absolutely continuous with respect to the surface measure in a suitably chosen intrinsic geometry. The results in Sections 8 and 9 are obtained by combining Harnack chains and Carleson estimates with explicit barrier constructions from Section 6 and decay estimates from Section 7.

Acknowledgment The first author was supported by the Swedish Research Council, dnr: 637-2014-6822. The second author was supported by the Academy of Finland \#258000.

\section{NOTATION AND PRELIMINARIES}

Points in $\mathbb{R}^{n+1}$ are denoted by $x=\left(x_{1}, \ldots, x_{n}, t\right)$. Given a set $E \subset \mathbb{R}^{n}$, let $\bar{E}, \partial E$, diam $E, E^{c}, E^{\circ}$, denote the closure, boundary, diameter, complement and interior of $E$, respectively. Let - denote the standard inner product on $\mathbb{R}^{n}$, let $|x|=(x \cdot x)^{1 / 2}$ be the Euclidean norm of $x$, and let $\mathrm{d} x$ be Lebesgue $n$-measure on $\mathbb{R}^{n}$. Given $x \in \mathbb{R}^{n}$ and $r>0$, let $B_{r}(x)=\left\{y \in \mathbb{R}^{n}:|x-y|<r\right\}$. Given $E, F \subset \mathbb{R}^{n}$, let $d(E, F)$ be the Euclidean distance from $E$ to $F$. In case $E=\{y\}$, we write $d(y, F)$. For simplicity, we define sup to be the essential supremum and inf to be the essential infimum. If $O \subset \mathbb{R}^{n}$ is open and $1 \leq q \leq \infty$, then by $W^{1, q}(O)$ we denote the space of equivalence classes of functions $f$ with distributional gradient $\nabla f=\left(f_{x_{1}}, \ldots, f_{x_{n}}\right)$, both of which are $q$-th power integrable on $O$. Let

$$
\|f\|_{W^{1, q}(O)}=\|f\|_{L^{q}(O)}+\||\nabla f|\|_{L^{q}(O)}
$$

be the norm in $W^{1, q}(O)$ where $\|\cdot\|_{L^{q}(O)}$ denotes the usual Lebesgue $q$-norm in $O$. $C_{0}^{\infty}(O)$ is the set of infinitely differentiable functions with compact support in $O$ and we let $W_{0}^{1, q}(O)$ denote the closure of $C_{0}^{\infty}(O)$ in the norm $\|\cdot\|_{W^{1, q}(O)} \cdot W_{\text {loc }}^{1, q}(O)$ is defined in the standard way. By $\nabla \cdot$ we denote the divergence operator. Given $t_{1}<t_{2}$ we denote by $L^{q}\left(t_{1}, t_{2}, W^{1, q}(O)\right)$ the space of functions such that for almost 
every $t, t_{1} \leq t \leq t_{2}$, the function $x \rightarrow u(x, t)$ belongs to $W^{1, q}(O)$ and

$$
\|u\|_{L^{q}\left(t_{1}, t_{2}, W^{1, q}(O)\right)}:=\left(\int_{t_{1}}^{t_{2}} \int_{O}\left(|u(x, t)|^{q}+|\nabla u(x, t)|^{q}\right) \mathrm{d} x \mathrm{~d} t\right)^{1 / q}<\infty .
$$

The spaces $L^{q}\left(t_{1}, t_{2}, W_{0}^{1, q}(O)\right)$ and $L_{\text {loc }}^{q}\left(t_{1}, t_{2}, W_{\text {loc }}^{1, q}(O)\right)$ are defined analogously. Finally, for $I \subset \mathbb{R}$, we denote $C\left(I ; L^{q}(O)\right)$ as the space of functions such that $t \rightarrow$ $\|u(t, \cdot)\|_{L^{q}(O)}$ is continuous whenever $t \in I$. $C_{\mathrm{loc}}\left(I ; L_{\mathrm{loc}}^{q}(O)\right)$ is defined analogously.

2.1. Weak solutions. Let $\Omega \subset \mathbb{R}^{n}$ be a bounded domain, i.e., a connected open set. For $t_{1}<t_{2}$, we let $\Omega_{t_{1}, t_{2}}:=\Omega \times\left(t_{1}, t_{2}\right)$. Given $p, 1<p<\infty$, we say that $u$ is a weak solution to

$$
\partial_{t} u-\Delta_{p} u=0
$$

in $\Omega_{t_{1}, t_{2}}$ if $u \in L_{\mathrm{loc}}^{p}\left(t_{1}, t_{2}, W_{\mathrm{loc}}^{1, p}(\Omega)\right)$ and

$$
\int_{\Omega_{t_{1}, t_{2}}}\left(-u \partial_{t} \phi+|\nabla u|^{p-2} \nabla u \cdot \nabla \phi\right) \mathrm{d} x \mathrm{~d} t=0
$$

whenever $\phi \in C_{0}^{\infty}\left(\Omega_{t_{1}, t_{2}}\right)$. If $u$ is a weak solution to (2.1) in the above sense, then we will often refer to $u$ as being $p$-parabolic in $\Omega_{t_{1}, t_{2}}$. For $p \in(2, \infty)$ we have by the parabolic regularity theory, see [9], that any $p$-parabolic function $u$ has a locally Hölder continuous representative. In particular, in the following we will assume that $p \in(2, \infty)$ and any solution $u$ is continuous. If $(2.2)$ holds with $=$ replaced by $\geq(\leq)$ for all $\phi \in C_{0}^{\infty}\left(\Omega_{t_{1}, t_{2}}\right), \phi \geq 0$, then we will refer to $u$ as a weak supersolution (subsolution).

2.2. Geometry. We here state the geometrical notions used throughout the paper.

Definition 2.1. A bounded domain $\Omega$ is called non-tangentially accessible (NTA) if there exist $M \geq 2$ and $r_{0}$ such that the following are fulfilled:

(1) corkscrew condition: for any $w \in \partial \Omega, 0<r<r_{0}$, there exists a point $a_{r}(w) \epsilon$ $\Omega$ such that

$$
M^{-1} r<\left|a_{r}(w)-w\right|<r, \quad d\left(a_{r}(w), \partial \Omega\right)>M^{-1} r,
$$

(2) $\mathbb{R}^{n} \backslash \Omega$ satisfies (1),

(3) uniform condition: if $w \in \partial \Omega, 0<r<r_{0}$, and $w_{1}, w_{2} \in B_{r}(w) \cap \Omega$, then there exists a rectifiable curve $\gamma:[0,1] \rightarrow \Omega$ with $\gamma(0)=w_{1}, \gamma(1)=w_{2}$, such that (a) $H^{1}(\gamma) \leq M\left|w_{1}-w_{2}\right|$,

(b) $\min \left\{H^{1}(\gamma([0, t])), H^{1}(\gamma([t, 1]))\right\} \leq M d(\gamma(t), \partial \Omega)$, for all $t \in[0,1]$.

We choose this definition as it very useful when we explicitly construct the parabolic Harnack chains in Section 3, see specifically Theorem 3.5. The values $M$ and $r_{0}$ will be called the NTA-constants of $\Omega$. For more on the notion of NTA-domains we refer to [18].

Definition 2.2. Let $\Omega \subset \mathbb{R}^{n}$ be a bounded domain. We say that $\Omega$ satisfies the ball condition with radius $r_{0}>0$ if for each point $y \in \partial \Omega$ there exists points $x^{+} \in \Omega$ and $x^{-} \in \Omega^{c}$ such that $B_{r_{0}}\left(x^{+}\right) \subset \Omega, B_{r_{0}}\left(x^{-}\right) \subset \Omega^{c}, \partial B_{r_{0}}\left(x^{+}\right) \cap \partial \Omega=\{y\}=\partial B_{r_{0}}\left(x^{-}\right) \cap \partial \Omega$, and such that the points $x^{+}(y), x^{-}(y), y$ are collinear for each $y \in \partial \Omega$.

Remark 2.3. It is easy to see that a domain satisfying the ball condition with radius $r_{0}>0$ is an NTA-domain with a constant $M$ and $r_{0}$. In particular, we may canonically choose

$$
a_{r}\left(x_{0}\right):=x_{0}+\frac{r}{2} \frac{x^{+}-x_{0}}{\left|x^{+}-x_{0}\right|},
$$

since the direction given by $\frac{x^{+}-x_{0}}{\left|x^{+}-x_{0}\right|}$ is unique. The exterior corkscrew point is defined analogously. 
Remark 2.4. Let $\Omega \subset \mathbb{R}^{n}$ be a bounded domain. Then $\Omega$ is a $C^{1,1}$ domain if and only if it satisfies the ball condition. For a proof of this fact, see for example $[1$, Lemma 2.2].

2.3. The continuous Dirichlet problem. Assuming that $\Omega$ is a bounded NTAdomain one can prove, see [7] and [20], that all points on the parabolic boundary

$$
\partial_{p} \Omega_{T}=S_{T} \cup(\bar{\Omega} \times\{0\}), \quad S_{T}=\partial \Omega \times[0, T],
$$

of the cylinder $\Omega_{T}$ are regular for the Dirichlet problem for the equation (2.1). In particular, for any $f \in C\left(\partial_{p} \Omega_{T}\right)$, there exists a unique Perron-solution $u=u_{f}^{\Omega_{T}} \epsilon$ $C\left(\bar{\Omega}_{T}\right)$ to the Dirichlet problem $\partial_{t} u-\Delta_{p} u=0$ in $\Omega_{T}$ and $u=f$ on $\partial_{p} \Omega_{T}$.

\section{HARNACK CHAINS}

In this section we prove a sequence of results concerning intrinsic Harnack chains. Forward-in-time chains describe the diffusion with an appropriate waiting time. On the other hand, backward-in-time chains says that if the solution has existed for long enough time, the future values will control the values from the past as well. Throughout the section we let $\Omega \subset \mathbb{R}^{n}$ be a bounded domain and given $T>0$ we let $\Omega_{T}=\Omega \times(0, T)$.

3.1. Local Harnack inequalities. We here collect two estimates from the literature. The following theorem can be found in $[9,10,11]$.

Theorem 3.1. Let $u$ be a non-negative $p$-parabolic function in $\Omega_{T}$, let $\left(x_{0}, t_{0}\right) \in \Omega_{T}$ and assume that $u\left(x_{0}, t_{0}\right)>0$. There exist positive constants $c_{h}$ and $C_{h}$, depending only on $p, n$, such that if $B_{4 r}\left(x_{0}\right) \subset \Omega$ and

$$
\left(t_{0}-\theta(4 r)^{p}, t_{0}+\theta(4 r)^{p}\right) \subset(0, T]
$$

where $\theta=\left(\frac{c_{h}}{u\left(x_{0}, t_{0}\right)}\right)^{p-2}$, then

$$
u\left(x_{0}, t_{0}\right) \leq C_{h} \inf _{B_{r}\left(x_{0}\right)} u\left(\cdot, t_{0}+\theta r^{p}\right) .
$$

The constants $c_{h}$ and $C_{h}$ are stable as $p \rightarrow 2$ and deteriorate as $p \rightarrow \infty$ in the sense that $c_{h}(p), C_{h}(p) \rightarrow \infty$ as $p \rightarrow \infty$.

The next theorem is instead valid for non-negative weak supersolutions. For the proof we refer to [22].

Theorem 3.2. Let $u$ be a non-negative weak supersolution in $B_{4 r}\left(x_{0}\right) \times(0, T)$. There exist constants $C_{i} \equiv C_{i}(p, n), i=1,2$, such that

$$
f_{B_{r}\left(x_{0}\right)} u\left(x, t_{1}\right) \mathrm{d} x \leq \frac{1}{2}\left(\frac{C_{1} r^{p}}{T-t_{1}}\right)^{\frac{1}{p-2}}+C_{2} \inf _{Q} u
$$

for almost every $0<t_{1}<T$, where $Q:=B_{2 r}\left(x_{0}\right) \times\left(t_{1}+T_{1} / 2, t_{1}+T_{1}\right)$, and

$$
T_{1}=\min \left\{T-t_{1}, C_{1} r^{p}\left(f_{B_{r}\left(x_{0}\right)} u\left(x, t_{1}\right) \mathrm{d} x\right)^{2-p}\right\} .
$$

In particular, if $T_{1}<T-t_{1}$, then

$$
f_{B_{r}\left(x_{0}\right)} u\left(x, t_{1}\right) \mathrm{d} x \leq 2 C_{2} \inf _{Q} u .
$$


3.2. Forward Harnack chains. We begin by describing a simple Harnack chain for weak supersolutions.

Lemma 3.3 (Weak forward Harnack chains). Let $\Omega \subset \mathbb{R}^{n}$ be a domain and let $T>0$. Let $x, y$ be two points in $\Omega$ and assume that there exist a sequence of balls $\left\{B_{4 r}\left(x_{j}\right)\right\}_{j=0}^{k}$ such that $x_{0}=x, x_{k}=y, B_{4 r}\left(x_{j}\right) \subset \Omega$ for all $j=0, \ldots, k$ and that $x_{j+1} \in B_{r}\left(x_{j}\right), j=0, \ldots, k-1$. Assume that $u$ is a continuous non-negative weak supersolution in $\Omega_{T}$ with

$$
\bar{\Lambda}:=f_{B_{r}\left(x_{0}\right)} u\left(x, t_{0}\right) \mathrm{d} x>0 .
$$

There exist constants $\bar{c}_{i} \equiv \bar{c}_{i}(p, n)>1, i \in\{1,2\}$, such that if

$$
t_{0}+\tau_{k} \bar{\Lambda}^{2-p} r^{p}<T, \quad \tau_{k}:=\bar{c}_{1} \sum_{j=0}^{k} \bar{c}_{2}^{j(p-2)},
$$

then

$$
f_{B_{r}(x)} u\left(x, t_{0}\right) \mathrm{d} x \leq \bar{c}_{2}^{k+1} \inf _{z \in B_{2 r}(y)} u\left(z, t_{0}+\tau_{k} \bar{\Lambda}^{2-p} r^{p}\right) .
$$

Furthermore, constants $\bar{c}_{i}, i \in\{1,2\}$, are stable as $p \rightarrow 2^{+}$. In particular, when $p=2$, then $\tau_{k}=\bar{c}_{1}(k+1)$ with $\bar{c}_{1}=\bar{c}_{1}(n)$.

Proof. Using Theorem 3.2 we first get that

$$
\frac{\bar{\Lambda}}{2 C_{2}} \leq \inf _{z \in B_{2 r}\left(x_{0}\right)} u\left(z, t_{1}\right), \quad t_{1}:=t_{0}+C_{1} \bar{\Lambda}^{2-p} r^{p} .
$$

Define then

$$
u_{j}:=\min \left(u, \Lambda_{j}\right), \quad \Lambda_{j}:=\left(2 C_{2}\right)^{-j} \bar{\Lambda}, \quad t_{j+1}:=t_{j}+C_{1} \Lambda_{j}^{2-p} r^{p},
$$

for $j=\{1, \ldots, k-1\}$. Assume inductively, that for $t_{i+1} \leq T$ we have

$$
B_{r}\left(x_{i}\right) \subset B_{2 r}\left(x_{i-1}\right),
$$

and

$$
u_{i}\left(z, t_{i}\right)=\Lambda_{i} \quad \text { for } z \in B_{r}\left(x_{i}\right),
$$

hold for $i \in\{0, \ldots, j\}$. For $j=1$ this is certainly the case as we see from (3.1). Since $u_{j}$ is a non-negative weak supersolution, Theorem 3.2 gives us

$$
\Lambda_{j+1}=\frac{\Lambda_{j}}{2 C_{2}} \leq \inf _{z \in B_{2 r}\left(x_{j}\right)} u_{j}\left(z, t_{j+1}\right),
$$

and hence also

$$
u_{j+1}\left(z, t_{j+1}\right)=\Lambda_{j+1} \quad \text { for } z \in B_{r}\left(x_{j+1}\right) .
$$

This proves the induction step. By the construction

$$
\inf _{z \in B_{r}(y)} u_{k}\left(z, t_{k}\right)=\Lambda_{k}
$$

holds. Thus, applying Theorem 3.2 one more time we get

$$
\inf _{z \in B_{2 r}(y)} u(z, \bar{t}) \geq\left(2 C_{2}\right)^{-(k+1)} \bar{\Lambda}
$$

with

$$
\bar{t}:=t_{0}+C_{1} \sum_{j=0}^{k}\left(2 C_{2}\right)^{j(p-2)} \bar{\Lambda}^{2-p} r^{p} .
$$

Setting $\bar{c}_{1}=C_{1}$ and $\bar{c}_{2}=2 C_{2}$ completes the proof of the lemma.

For $p$-parabolic functions we have the following pointwise version of Lemma 3.3. 
Proposition 3.4. Let $\Omega \subset \mathbb{R}^{n}$ be a domain and let $T>0$. Let $x, y$ be two points in $\Omega$ and assume that there exist a sequence of balls $\left\{B_{4 r}\left(x_{j}\right)\right\}_{j=0}^{k}$ such that $x_{0}=x, x_{k}=y$, $B_{4 r}\left(x_{j}\right) \subset \Omega$ for all $j=0, \ldots, k$ and that $x_{j+1} \in B_{r}\left(x_{j}\right), j=0, \ldots, k-1$. Assume that $u$ is a non-negative p-parabolic function in $\Omega_{T}$ and assume that $u\left(x, t_{0}\right)>0$. There exist constants $c \equiv c(p, n)$ and $c_{1} \equiv c_{1}(p, n, k)>1$ such that if

$$
t_{0}-\left(c_{h} / u\left(x, t_{0}\right)\right)^{p-2}(4 r)^{p}>0, t_{0}+c_{1}(k) u\left(x, t_{0}\right)^{2-p} r^{p}<T,
$$

then

$$
u\left(x, t_{0}\right) \leq c^{k} \inf _{z \in B_{r}(y)} u\left(z, t_{0}+c_{1}(k) u\left(x, t_{0}\right)^{2-p} r^{p}\right) .
$$

Furthermore, $c_{1}$ satisfies the estimate

$$
\widetilde{c}_{1} k \leq c_{1} \leq \widetilde{c}_{1}(k+1) c^{(k+1)(p-2)}
$$

with $\widetilde{c}_{1} \equiv \widetilde{c}_{1}(p, n)$ and $\widetilde{c}_{1}(p, n) \rightarrow \widetilde{c}_{1}(n)$ as $p \rightarrow 2$.

Proof. After applying Theorem 3.1 once, the result follows from Lemma 3.3.

We next focus on cylindrical NTA-domains. The first theorem, Theorem 3.5, holds for weak supersolutions and shows how to bound the values of a solution at points close to the boundary using pointwise interior values. A remarkable fact of the proof is that the waiting time is explicitly defined and, as $p \rightarrow 2$, it gives a supersolution version of the theorem of Salsa [31, Theorem C] as alluded to in the introduction, see (1.5). The proof uses heavily the assumptions on NTA-domains and iterations of Lemma 3.3.

Theorem 3.5. Let $\Omega \subset \mathbb{R}^{n}$ be an NTA-domain with constants $M$ and $r_{0}$, let $x_{0} \in$ $\partial \Omega, T>0$ and let $0<r<r_{0}$. Let $x, y$ be two points in $\Omega \cap B_{r}\left(x_{0}\right)$ such that

$$
\varrho:=d(x, \partial \Omega) \leq r \quad \text { and } \quad d(y, \partial \Omega) \geq \frac{r}{4} .
$$

Assume that $u$ is a non-negative continuous weak supersolution in $\Omega_{T}$, and assume that

$$
\Lambda:=f_{B_{\varrho / 4}(x)} u\left(z, t_{0}\right) \mathrm{d} z>0 .
$$

Let $\delta \in(0,1]$. Then there exist positive constants $c_{i} \equiv c_{i}(M, p, n), i \in\{1,2,3\}$, such that if $t_{0}+\tau<T$, where

$$
\tau:=\delta^{p-1}\left(c_{2}^{-1 / \delta}\left(\frac{r}{\varrho}\right)^{-c_{3} / \delta} \Lambda\right)^{2-p} r^{p}
$$

then

$$
f_{B_{\varrho / 4}(x)} u\left(z, t_{0}\right) \mathrm{d} z \leq c_{1}^{1 / \delta}\left(\frac{r}{\varrho}\right)^{c_{3} / \delta} \inf _{z \in B_{r / 16}(y)} u\left(z, t_{0}+\tau\right) .
$$

Furthermore, constants $c_{i}, i \in\{1,2,3\}$, are stable as $p \rightarrow 2^{+}$.

Proof. We split the proof into three steps.

Step 1: Parametrization of the curve connecting $x$ and $y$. According to the uniform condition (3) in Definition 2.1, we can find a rectifiable curve $\gamma$ connecting $x$ and $y$ such that $\gamma(0)=x, \gamma(1)=y$, and

(a) $H^{1}(\gamma) \leq M\left|w_{1}-w_{2}\right|$,

(b) $\min \left\{H^{1}(\gamma([0, t])), H^{1}(\gamma([t, 1]))\right\} \leq M d(\gamma(t), \partial \Omega)$, for all $t \in[0,1]$. 
We call a ball $B \subset \Omega$ admissible if $4 B \subset \Omega$, and is thus eligible for the Harnack inequality. Our goal with with step is to construct a sequence of admissible balls covering the curve $\gamma$. In the following we may, without loss of generality, assume that $H^{1}(\gamma([0,1]))>2^{-4} r$. We define $\hat{t}_{1}, \hat{t}_{2} \in(0,1)$ such that $H^{1}\left(\gamma\left(\left[0, \hat{t}_{1}\right]\right)\right)=2^{-5} r$ and $H^{1}\left(\gamma\left(\left[\hat{t}_{2}, 1\right]\right)\right)=2^{-5} r$. The technical part will be in the interval $\left(0, \hat{t}_{1}\right)$. To continue we choose $k$ as the integer which satisfies $2^{-k} r \in(\varrho / 16, \varrho / 8]$. We define sequence of real number $\left\{s_{j}\right\}$ through

$$
H^{1}\left(\gamma\left(\left[0, s_{j}\right]\right)\right)=2^{-k+j} H^{1}\left(\gamma\left(\left[0, \hat{t}_{1}\right]\right)\right):=2^{-k-5+j} r, \quad s_{0}=0 .
$$

Then, for any $s \in\left[s_{j}, s_{j+1}\right)$, (a) and (b) implies

$$
d(\gamma(s), \partial \Omega) \geq \frac{2^{-k+j-5} r}{M}
$$

and

$$
H^{1}\left(\gamma\left(\left[s_{j}, s_{j+1}\right]\right)\right)=2^{-k+j-5} r
$$

Thus, defining

$$
\varrho_{j}:=N^{-1} 2^{-k+j-5} r, \quad N \in \mathbb{N}, \quad N \geq 2^{9} M,
$$

we see that the piece $\gamma\left(\left[s_{j}, s_{j+1}\right]\right)$ can be covered with $N$ admissible balls of the type $B^{i, j}:=B_{\varrho_{j}}\left(y_{i, j}\right)$ such that $y_{i, j} \in \gamma\left(\left[s_{j}, s_{j+1}\right]\right)$ for $i \in\{1, \ldots, N\}, y_{i, j-1} \in B^{i, j}$ and $\gamma\left(\left[s_{j}, s_{j+1}\right]\right) \subset \cup_{i} B^{i, j}$. Finally, we observe that the middle piece of the curve $\gamma\left(\left[\hat{t}_{1}, \hat{t}_{2}\right]\right)$, due to the definition of $\hat{t}_{1}, \hat{t}_{2}$ together with (b) can be covered with $M N$ admissible balls of size $r / N$. Moreover we can cover the end piece $\gamma\left(\left[\hat{t}_{2}, 1\right]\right)$ with $N$ admissible balls of size $r / N$ since $\gamma\left(\left[\hat{t}_{2}, 1\right]\right) \subset B_{r / 16}(y) \subset \Omega$. At this point, we consider $N \in \mathbb{N}$ to be a free parameter such that $N \geq 2^{9} M$.

Step 2: Iteration via Harnack estimates. Let now $\Lambda$ be as in (3.2). Theorem 3.2 implies that if

$$
t_{0}+C_{1} \Lambda^{2-p} \varrho_{0}^{p}<T
$$

then

$$
\inf _{z \in B_{\varrho_{0}}\left(x_{0}\right)} u\left(z, t_{1}\right) \geq \frac{1}{2 C_{2}} \Lambda, \quad t_{1}:=t_{0}+C_{1} \Lambda^{2-p} \varrho_{0}^{p} .
$$

Let

$$
\Lambda_{1}=\sigma \Lambda, \quad \sigma \in\left(0,\left(2 C_{2}\right)^{-1}\right] .
$$

Defining thus $u_{1}:=\min \left(u, \Lambda_{1}\right)$, we obtain by Lemma 3.3 (see also the proof of that Lemma) that there exist constants $\bar{c}_{1} \equiv \bar{c}_{1}(p, n)$ and $\bar{c}_{2} \equiv \bar{c}_{2}(p, n)$, such that if

$$
t_{2}:=t_{1}+\tau_{N} \Lambda_{1}^{2-p} \varrho_{1}^{p}<T, \quad \tau_{N}:=\bar{c}_{1} \sum_{j=0}^{N-1} \bar{c}_{2}^{j(p-2)} \in\left[\bar{c}_{1} N, \bar{c}_{1} N \bar{c}_{2}^{N(p-2)}\right)
$$

then

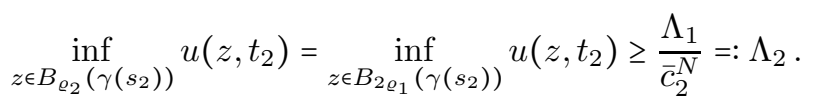

Define

$$
\Lambda_{j+1}:=\bar{c}_{2}^{-(j+1) N} \Lambda_{1}, \quad u_{j}:=\min \left(u, \Lambda_{j}\right), \quad j \in \mathbb{N},
$$

let $\hat{k}:=k+M+1$, and let

$$
t_{j+1}:= \begin{cases}t_{j}+\tau_{N} \Lambda_{j}^{2-p} \varrho_{j}^{p} & \text { if } j \in\{1, \ldots, k\} \\ t_{j}+\tau_{N} \Lambda_{j}^{2-p}(r / N)^{p} & \text { if } j \in\{k+1, \ldots, \hat{k}\}\end{cases}
$$

Iterating Lemma 3.3 it follows by induction that

$$
u\left(y, t_{\hat{k}+1}\right) \geq \Lambda_{\hat{k}+1} .
$$


Step 3: Waiting time. Let us now analyze the waiting time $t_{\hat{k}+1}$, which we want to show to be precisely $t_{0}+\tau$ by a suitable choice of $\Lambda_{1}$. We have

$$
\begin{aligned}
t_{\hat{k}+1}=t_{1} & +C_{1} \Lambda^{2-p} \varrho_{0}^{p}+\tau_{N}\left[\sum_{j=1}^{k} \Lambda_{j}^{2-p} \varrho_{j}^{p}+\sum_{j=k+1}^{k} \Lambda_{j}^{2-p} \varrho_{j}^{p}\right] \\
=t_{0} & +C_{1} \Lambda^{2-p} \varrho_{0}^{p}+N^{-p} \tau_{N} \Lambda_{1}^{2-p} r^{p}\left[2^{-k p} \sum_{j=0}^{k}\left(2^{p} \bar{c}_{2}^{(p-2) N}\right)^{j}\right. \\
& \left.+\bar{c}_{2}^{(p-2) k N} \sum_{j=0}^{M}\left(\bar{c}_{2}^{(p-2) N}\right)^{j}\right] \\
=: & t_{0}+\mathbf{T} \Lambda^{2-p} r^{p} .
\end{aligned}
$$

We can write the sum in (3.5) as follows

$$
2^{-k p} \sum_{j=0}^{k}\left(2^{p} \bar{c}_{2}^{(p-2) N}\right)^{j}=2^{p} \frac{\bar{c}_{2}^{(p-2)(k+1) N}-2^{-(k+1) p}}{2^{p} \bar{c}_{2}^{(p-2) N}-1},
$$

while the sum in (3.6) can be estimated similarly to $\tau_{N}$ (see (3.3))

$$
\bar{c}_{2}^{(p-2) k N} \sum_{j=0}^{M}\left(\bar{c}_{2}^{(p-2) N}\right)^{j} \in\left(M \bar{c}_{2}^{(p-2) k N}, M \bar{c}_{2}^{(p-2)(k+M) N}\right] .
$$

Hence, recalling the definition of $\tau_{N}$ and $\mathbf{T}$, we get after some straightforward estimation that

$$
\frac{1}{2} \bar{c}_{1} \sigma^{2-p} N^{1-p}\left(\frac{r}{\varrho}\right)^{(p-2) \bar{c}_{4} N} \leq \mathbf{T} \leq 2 \bar{c}_{3} \sigma^{2-p} N^{1-p} \bar{c}_{3}^{(p-2) N}\left(\frac{r}{\varrho}\right)^{(p-2) \bar{c}_{4} N}
$$

for new constants $\bar{c}_{3}, \bar{c}_{4}$ depending only on $p, n, M$. We now choose $N=\widetilde{c} / \delta$ and let $\widetilde{c}$ be a degree of freedom. First note that choosing $\sigma_{1}=\left(2 C_{2}\right)^{-1}$, then choose $c_{3}=\widetilde{c} c_{4}$ and $c_{2}=\left[\bar{c}_{3}\right]^{\widetilde{c}}$, then for a large enough $\widetilde{c}=\widetilde{c}(p, n, M)$

$$
2 \bar{c}_{3}\left(2 C_{2}\right)^{p-2} \widetilde{c}^{1-p} \bar{c}_{3}^{(p-2) \widetilde{c} / \delta}\left(\frac{r}{\varrho}\right)^{(p-2) \bar{c}_{4} \widetilde{c} / \delta}<c_{2}^{(p-2) / \delta}\left(\frac{r}{\varrho}\right)^{(p-2) c_{3} / \delta} .
$$

Second we see that choosing $\sigma_{2}=c_{5}^{-1 / \delta}$ for large enough $c_{5}=c_{5}(p, n, M)$ the following holds

$$
\frac{1}{2} \bar{c}_{1} \sigma_{2}^{2-p} \widetilde{c}^{1-p}\left(\frac{r}{\varrho}\right)^{(p-2) \bar{c}_{4} \widetilde{c} / \delta}>c_{2}^{(p-2) / \delta}\left(\frac{r}{\varrho}\right)^{(p-2) c_{3} / \delta} .
$$

With (3.7)-(3.9) at hand we see that there is a choice of $\sigma \in\left[\sigma_{2}, \sigma_{1}\right]$ such that

$$
\mathbf{T}=\delta^{p-1} c_{2}^{(p-2) / \delta}\left(\frac{r}{\varrho}\right)^{(p-2) c_{3} / \delta},
$$

and thus we have proved $t_{\hat{k}+1}=t_{0}+\mathbf{T} \Lambda^{2-p} r^{p}=t_{0}+\tau$. This together with (3.4) finishes the proof by taking suitably large $c_{1}$ in the statement.

For $p$-parabolic functions we have the following pointwise version of Theorem 3.5.

Theorem 3.6. Let $\Omega \subset \mathbb{R}^{n}$ be an NTA-domain with constants $M$ and $r_{0}$, let $x_{0} \in$ $\partial \Omega, T>0$ and let $0<r<r_{0}$. Let $x, y$ be two points in $\Omega \cap B_{r}\left(x_{0}\right)$ such that

$$
\varrho:=d(x, \partial \Omega) \leq r \quad \text { and } \quad d(y, \partial \Omega) \geq \frac{r}{4} .
$$

Assume that $u$ is a non-negative p-parabolic function in $\Omega_{T}$, and assume that $u\left(x, t_{0}\right)$ is positive. Let $\delta \in(0,1]$. Then there exist constants $c_{i} \equiv c_{i}(M, p, n)$, $i \in\{1,2,3\}$, such that if

$$
t_{0}-\left(c_{h} / u\left(x, t_{0}\right)\right)^{p-2}(\delta \varrho)^{p}>0, \quad t_{0}+\tau<T,
$$


with

$$
\tau:=\delta^{p-1}\left(c_{2}^{-1 / \delta}\left(\frac{r}{\varrho}\right)^{-c_{3} / \delta} u\left(x, t_{0}\right)\right)^{2-p} r^{p},
$$

then

$$
u\left(x, t_{0}\right) \leq c_{1}^{1 / \delta}\left(\frac{r}{\varrho}\right)^{c_{3} / \delta} \inf _{z \in B_{r / 16}(y)} u\left(z, t_{0}+\tau\right) .
$$

Furthermore, constants $c_{i}, i \in\{1,2,3\}$, are stable as $p \rightarrow 2^{+}$.

Proof. Applying Theorem 3.1 once, we see that the theorem follows from Theorem 3.5.

3.3. Backward Harnack chains. The philosophy of the forward Harnack chains in Section 3.2 is that the data at the starting point will start to diffuse according to the intrinsic Harnack inequality. The finite speed of diffusion forces the waiting time to blow up if we wish to spread our information in an infinite chain. In the backward Harnack chains that we develop in this section the philosophy is reversed. Instead of looking to the future we look to the past. This means that if the value of the solution at a point $(y, s)$ is, say, one, then we ask the question: how large can the values in the past be without violating the fact that the solution is one at $(y, s)$.

We start with the weak version of the backward Harnack chains, valid for weak supersolutions.

Theorem 3.7. Let $\Omega \subset \mathbb{R}^{n}$ be an NTA-domain with with constants $M$ and $r_{0}$, let $x_{0} \in \partial \Omega, T>0$, and let $0<r<r_{0}$. Let $x, y$ be two points in $\Omega \cap B_{r}\left(x_{0}\right)$ such that

$$
\varrho:=d(x, \partial \Omega) \leq r \quad \text { and } \quad d(y, \partial \Omega) \geq \frac{r}{4} .
$$

Assume that $u$ is a non-negative continuous weak supersolution in $\Omega_{T}$, and assume that $u(y, s)$ is positive. Let $\delta \in(0,1]$. Then there exist positive constants $C_{i} \equiv$ $C_{i}(p, n)$ and $c_{i} \equiv c_{i}(p, n, M), i \in\{4,5\}$, such that if $s \in(\tau, T)$ and

$$
t \in\left[s-\tau, s-\delta^{p-1} \tau\right],
$$

with

$$
\tau:=C_{4}\left[C_{5} u(y, s)\right]^{2-p} r^{p}
$$

then

$$
f_{B_{\varrho / 4}(x)} u(z, t) \mathrm{d} z \leq c_{4}^{1 / \delta}\left(\frac{r}{\varrho}\right)^{c_{5} / \delta} u(y, s) .
$$

Furthermore, constants $c_{i}, C_{i}, i \in\{4,5\}$, are stable as $p \rightarrow 2^{+}$.

Remark 3.8. If we assume that $u \in C\left([0, T) ; L^{2}(\Omega)\right)$ then we can replace $s \in(\tau, T)$ with $s \in[\tau, T)$. That is, the chain can be taken all the way to the initial time, i.e. $t=0$.

Proof. After scaling, we may assume that $u(y, s)=1$. Assume now the contrary to (3.10), i.e.

$$
f_{B_{\varrho / 4}(x)} u(z, t) \mathrm{d} z>H\left(\frac{r}{\varrho}\right)^{c_{5} / \delta}
$$

for constants $c_{5}, H$ to be fixed. Then Theorem 3.5 implies that with

$$
\widetilde{\tau}:=\widetilde{\delta}^{p-1}\left(c_{2}^{-1 / \widetilde{\delta}}\left(\frac{r}{\varrho}\right)^{-c_{3} / \widetilde{\delta}} f_{B_{\varrho / 4}(x)} u(z, t) \mathrm{d} z\right)^{2-p} r^{p}
$$


we get

$$
f_{B_{\varrho / 4}(x)} u(z, t) \mathrm{d} z \leq c_{1}^{1 / \widetilde{\delta}}\left(\frac{r}{\varrho}\right)^{c_{3} / \widetilde{\delta}} \inf _{z \in B_{r / 16}(y)} u(z, t+\widetilde{\tau})
$$

with constants $c_{i} \equiv c_{i}(p, n, M), i \in\{1,2,3\}$ and $\widetilde{\delta} \in(0, \delta]$. Now we have an upper bound for $\widetilde{\tau}$ by means of (3.11) as follows:

$$
\begin{aligned}
\widetilde{\tau} & \leq \widetilde{\delta}^{p-1}\left(c_{2}^{-1 / \widetilde{\delta}}\left(\frac{r}{\varrho}\right)^{-c_{3} / \widetilde{\delta}} H\left(\frac{r}{\varrho}\right)^{c_{5} / \delta}\right)^{2-p} r^{p} \\
& =\widetilde{\delta}^{p-1}\left(c_{2}^{-1 / \widetilde{\delta}} H\right)^{2-p} r^{p} \\
& \leq \delta^{p-1} \tau,
\end{aligned}
$$

provided that

$$
H \geq C_{5} c_{2}^{1 / \widetilde{\delta}}, \quad \widetilde{\delta}:=\delta \min \left\{1, C_{4}\right\}^{1 /(p-1)}, \quad c_{5}:=\frac{c_{3}}{\min \left\{1, C_{4}\right\}^{1 /(p-1)}} .
$$

Therefore we have that

$$
t+\widetilde{\tau} \leq s .
$$

Observe that both $C_{4}$ and $C_{5}$ are still to be fixed. Thus we need to carry the information from the time $t+\widetilde{\tau}$ up to $s$. To this end, connecting (3.11) and (3.12) with the choices in (3.13) leads to

$$
H c_{1}^{-1 / \widetilde{\delta}}<\inf _{z \in B_{r / 16}(y)} u(z, t+\widetilde{\tau}) .
$$

Truncate $u$ as

$$
\widetilde{u}=\min \left(4 C_{2}, u\right)
$$

and take

$$
H:=c_{4}^{1 / \delta}, \quad c_{4}:=\max \left\{4 C_{2} c_{1}, C_{5} c_{2}\right\}^{1 / \min \left\{1, C_{4}\right\}^{1 /(p-1)}},
$$

where $C_{2}$ is as in Theorem 3.2. Then $\widetilde{u}$ is a continuous weak supersolution, and we have by (3.13)-(3.15) that

$$
f_{B_{\widetilde{r}}(y)} \widetilde{u}(z, t+\widetilde{\tau}) \mathrm{d} z=4 C_{2}, \quad \widetilde{r} \in(0, r / 16] .
$$

Applying thus the forward in time weak Harnack estimate in Theorem 3.2 gives

$$
4 C_{2} \leq 2 C_{2} \inf _{z \in B_{2 \widetilde{r}}(y)} \widetilde{u}\left(z, t+\widetilde{\tau}+C_{1}\left(4 C_{2}\right)^{2-p} \widetilde{r}^{p}\right)
$$

provided that

$$
t+\widetilde{\tau}+C_{1}\left(4 C_{2}\right)^{2-p} \widetilde{r}^{p}<T .
$$

Choosing $C_{4}=16^{-p} C_{1}$ and $C_{5}=4 C_{2}$, we always find $\widetilde{r} \leq r / 16$ such that

$$
t+\widetilde{\tau}+C_{1}\left(4 C_{2}\right)^{2-p} \widetilde{r}^{p}=s,
$$

and hence

$$
2 \leq \inf _{z \in B_{2 \widetilde{r}}(y)} \widetilde{u}(z, s) .
$$

This gives a contradiction since we assumed that $u(y, s)=1$, and thus the proof is complete.

The following theorem is the corresponding result for weak solutions, where we use pointwise information in the past instead of information in mean. The main difference this imposes on the assumptions in Theorem 3.7 is that we have to require the solution to have lived for a certain amount of time, which is precisely the price we have to pay if we wish to control pointwise values in the past. 
Theorem 3.9. Let $\Omega \subset \mathbb{R}^{n}$ be an NTA-domain with with constants $M$ and $r_{0}$, let $x_{0} \in \partial \Omega, T>0$, and let $0<r<r_{0}$. Let $x, y$ be two points in $\Omega \cap B_{r}\left(x_{0}\right)$ such that

$$
\varrho:=d(x, \partial \Omega) \leq r \quad \text { and } \quad d(y, \partial \Omega) \geq \frac{r}{4} .
$$

Assume that $u$ is a non-negative $p$-parabolic function in $\Omega_{T}$, and assume that $u(y, s)$ is positive. Let $\delta \in(0,1]$. Then there exist positive constants $C_{i} \equiv C_{i}(p, n)$ and $c_{i} \equiv c_{i}(p, n, M), i \in\{4,5\}$, such that if $s<T$ and

$$
\max \left\{\left(\frac{c_{4}^{1 / \delta}}{c_{h}}\left(\frac{r}{\varrho}\right)^{c_{5} / \delta} u(y, s)\right)^{2-p}(\delta \varrho)^{p}, s-\tau\right\} \leq t \leq s-\delta^{p-1} \tau,
$$

with

$$
\tau:=C_{4}\left[C_{5} u(y, s)\right]^{2-p} r^{p}
$$

then

$$
u(x, t) \leq c_{4}^{1 / \delta}\left(\frac{r}{\varrho}\right)^{c_{5} / \delta} u(y, s) .
$$

Furthermore, constants $c_{i}, C_{i}, i \in\{4,5\}$, are stable as $p \rightarrow 2^{+}$.

Proof. To prove the lemma we follow the same outline as the proof of Theorem 3.7, but instead of assuming the contrary assumption (3.11) we assume the contrary assumption that

$$
u(x, t)>H\left(\frac{r}{\varrho}\right)^{c_{5} / \delta},
$$

for some constants $c_{5}, H$ to be fixed. Applying Theorem 3.6 instead of Theorem 3.5 we get

$$
u(x, t) \leq c_{1}^{1 / \widetilde{\delta}}\left(\frac{r}{\varrho}\right)^{c_{3} / \widetilde{\delta}} \inf _{z \in B_{r / 16}(y)} u(z, t+\widetilde{\tau}) .
$$

Note that it is the usage of Theorem 3.6 which requires the assumption (3.16). The proof now follows repeating the remaining part of the proof of Theorem 3.7 essentially verbatim.

\section{Carleson estimate}

In this section we prove, using the improved Harnack chain estimate in Theorem 3.9, a flexible Carleson estimate valid in cylindrical NTA-domains. Versions of the Carleson estimate was originally proved, for equations of $p$-parabolic type in [3] in Lipschitz cylinders, and in [2] in certain time-dependent space-time domains. We begin with a standard oscillation decay lemma valid for weak subsolutions, see for example [9].

Lemma 4.1. Let $\Omega \subset \mathbb{R}^{n}$ be an NTA-domain with constants $M$ and $r_{0}$. Let $u$ be a non-negative, continuous weak subsolution in $\Omega_{T}$. Let $\left(x_{0}, t_{0}\right) \in S_{T}, 0<r<r_{0}$,

$$
Q_{r}^{\lambda}\left(x_{0}, t_{0}\right):=\left(B_{r}\left(x_{0}\right) \cap \Omega\right) \times\left(t_{0}-\lambda^{2-p} r^{p}, t_{0}\right) \subset \Omega_{T},
$$

and assume that $u$ vanishes continuously on $S_{T} \cap Q_{r}^{\lambda}\left(x_{0}, t_{0}\right)$ and that

$$
\sup _{Q_{r}^{\lambda}\left(x_{0}, t_{0}\right)} u \leq \lambda .
$$

Then there is a constant $\sigma$ depending only on $p, n, M$ such that $Q_{\sigma r}^{\lambda / 2}\left(x_{0}, t_{0}\right) \subset$ $Q_{r}^{\lambda}\left(x_{0}, t_{0}\right)$ and

$$
\sup _{Q_{\sigma r}^{\lambda / 2}\left(x_{0}, t_{0}\right)} u \leq \frac{\lambda}{2} .
$$


In particular, we have that

$$
\sup _{Q_{\sigma^{j} r_{r}}^{2^{-j}}\left(x_{0}, t_{0}\right)} u \leq 2^{-j} \lambda
$$

for any $j \in \mathbb{N}$.

The following theorem is usually referred to as a Carleson estimate. We want to point out that, compared to [3], not only does it hold for cylindrical NTA-domains, but also the formulation is more flexible for applications. In particular, we are able to adjust the waiting time, the height of the cylinder, and the distance to the initial boundary. All these parameters influence the constant in the inequality and a Gaussian type behavior is proved to be present.

Theorem 4.2. Let $\Omega \subset \mathbb{R}^{n}$ be an NTA-domain with constants $M$ and $r_{0}$. Let $u$ be a non-negative, weak solution in $\Omega_{T}$. Let $(x, t) \in S_{T}$ and $0<r<r_{0}$. Assume that $u\left(a_{r}(x), t\right)>0$ and let

$$
\tau=\frac{C_{4}}{4}\left[C_{5} u\left(a_{r}(x), t\right)\right]^{2-p} r^{p},
$$

where $C_{4}$ and $C_{5}$, both depending on $p, n$, are as in Theorem 3.9. Assume that $t>\left(\delta_{1}^{p-1}+\delta_{2}^{p-1}+2 \delta_{3}^{p-1}\right) \tau$ for $0<\delta_{1} \leq \delta_{3} \leq 1, \delta_{2} \in(0,1)$ and that for a given $\lambda \geq 0$, the function $(u-\lambda)_{+}$vanishes continuously on $S_{T} \cap B_{r}(x) \times\left(t-\left(\delta_{1}^{p-1}+\delta_{2}^{p-1}+\right.\right.$ $\left.\left.\delta_{3}^{p-1}\right) \tau, t-\delta_{1}^{p-1} \tau\right)$ from $\Omega_{T}$. Then there exist constants $c_{i} \equiv c_{i}(M, p, n), i \in\{6,7\}$, such that

where $Q:=B_{r}(x) \times\left(t-\left(\delta_{1}^{p-2}+\delta_{2}^{p-1}\right) \tau, t-\delta_{1}^{p-1} \tau\right)$. Furthermore, constants $c_{i}, i \in\{6,7\}$, are stable as $p \rightarrow 2^{+}$

Remark 4.3. Note that in the case $p=2$, Theorem 4.2 for $\lambda=0$ is equivalent to the estimate above by linearity. However, for $p>2$ this result extends the ones in $[2,3]$ if $\lambda>0$.

Proof. By scaling the function $u$ we can assume that $u\left(a_{r}(x), t\right)=1$, and replacing $\lambda$ with its scaled version. Consider the boxes

$$
\widetilde{Q}_{\nu}:=\left(\Omega \cap B_{r}(x)\right) \times\left(t-\left(\delta_{1}^{p-1}+\delta_{2}^{p-1}+\delta_{3}^{p-1}\right) \tau, t-\nu^{p-1} \tau\right), \quad \nu \in\left\{\delta_{1}, \delta_{3}\right\},
$$

and denote also

$$
Q_{r}^{\theta}(x, t):=\left(\Omega \cap B_{r}(x)\right) \times\left(t-\theta^{2-p} r^{p}, t\right), \quad \theta>0 .
$$

Observe that with the choice of $\tau$, we have by Theorem 3.9, for any $(y, s) \in \widetilde{Q}_{\nu}$ with $d(y, \partial \Omega) \leq r$ and $\nu \in\left\{\delta_{1}, \delta_{3}\right\}$, that

$$
u(y, s) \leq c_{8}^{1 / \nu}\left(\frac{r}{d(y, \partial \Omega)}\right)^{c_{9} / \nu}
$$

holds with $c_{8}, c_{9}$ depending only on $p, n, M$ (apply Theorem 3.9 with the choice $\delta:=\nu \min \left\{1, C_{4} / 2\right\}$ in order to guarantee the conditions in (3.16)).

We proceed by induction via a contradiction assumption. Assume that $P_{0}=$ $\left(x_{0}, t_{0}\right) \in Q$, where $Q$ is as in the statement, is such that $u\left(P_{0}\right)>H+\lambda$ for some large $H$ to be fixed. Assume then that we find inductively points $P_{j}=\left(x_{j}, t_{j}\right) \in \widetilde{Q}_{\hat{\delta}_{j}}$, where $\hat{\delta}_{j}=\delta_{3}$ if $t_{j} \leq t_{0}-\delta_{3}^{p-1} \tau / 2$ and $\hat{\delta}_{j}=\delta_{1}$ if $t_{j}>t_{0}-\delta_{3}^{p-1} \tau / 2$ for any $j \in \mathbb{N}$. Set $r_{j}:=d\left(x_{j}, \partial \Omega\right)$ and let $x_{j}^{\prime} \in \partial \Omega$ be such that $r_{j}=\left|x_{j}-x_{j}^{\prime}\right|$. Assume inductively that

$$
\left\{\begin{array}{l}
u\left(P_{j}\right)>2^{j} H+\lambda \text { and } \\
t-\left(\delta_{1}^{p-1}+\delta_{2}^{p-1}+\delta_{3}^{p-1}\right) \tau<t_{j} \leq t_{j-1} \leq t_{j}+\left(2^{j} H\right)^{2-p}\left(r_{j-1} / \sigma\right)^{p}
\end{array}\right.
$$


holds for all $j \in\{1, \ldots, k\}$, where $\sigma \equiv \sigma(p, n, M) \in(0,1)$ is as in Lemma 4.1. We then want to show that for large enough $H$ this continues to hold for $j=k+1$ as well.

To show the induction step, observe that (4.1) and the induction assumption (4.2) imply that

$$
2^{j} H+\lambda<u\left(P_{j}\right) \leq c_{8}^{1 / \hat{\delta}_{j}}\left(\frac{r}{r_{j}}\right)^{c_{9} / \hat{\delta}_{j}} \quad \Longrightarrow \quad r_{j} \leq\left(2^{j} H c_{8}^{-1 / \hat{\delta}_{j}}\right)^{-\hat{\delta}_{j} / c_{9}} .
$$

Fixing

$$
H:=\left(\frac{4 c_{8}^{p / c_{9}} c_{9}}{p \sigma^{p} \log 2} \frac{\tau}{r^{p} \delta_{3}^{p}}\right)^{c_{9} /\left[p \delta_{1}\right]}=:\left(c_{6} / \delta_{3}\right)^{c_{7} / \delta_{1}},
$$

we have after simple manipulations that

$$
r_{j}^{p} \leq \sigma^{p} \frac{\delta_{3}^{p-1} \tau}{4} 2^{-j \hat{\delta}_{j} p / c_{9}} \frac{\delta_{3} p \log 2}{c_{9}} \leq \sigma^{p} \frac{\delta_{3}^{p-1} \tau}{4} \frac{2^{-j \hat{\delta}_{j} p / c_{9}}}{\sum_{j=0}^{\infty} 2^{-j \delta_{3} p / c_{9}}} .
$$

In particular,

$$
\left(2^{k+1} H\right)^{2-p}\left(r_{k} / \sigma\right)^{p} \leq\left(r_{k} / \sigma\right)^{p} \leq \frac{\delta_{3}^{p-1} \tau}{4} .
$$

We hence set $K_{1}:=Q_{r_{k}}^{2^{k} H}\left(x_{k}^{\prime}, t_{k}\right)$ and $K_{2}:=Q_{r_{k} / \sigma}^{2^{k+1} H}\left(x_{k}^{\prime}, t_{k}\right)$, and we deduce, by the induction assumption and the estimate in previous display, that $K_{2} \subset \Omega_{T}$. Now, if

$$
\sup _{K_{2}}(u-\lambda)_{+} \leq 2^{k+1} H
$$

then, using that $(u-\lambda)_{+}$is a weak subsolution, Lemma 4.1 would imply that

$$
\sup _{K_{1}} u \leq 2^{k} H+\lambda
$$

which is a contradiction since $P_{k} \in \bar{K}_{1}$. Thus there is $P_{k+1} \in K_{2}$ such that

$$
u\left(P_{k+1}\right)>2^{k+1} H+\lambda \text {. }
$$

By the definition of $K_{2}$ and $P_{k+1}$, we must have that

$$
t_{k+1} \leq t_{k} \leq t_{k+1}+\left(2^{k+1} H\right)^{2-p}\left(r_{k} / \sigma\right)^{p} .
$$

Therefore we are left to show that $t_{k+1} \geq t-\left(\delta_{1}^{p-1}+\delta_{2}^{p-1}+\delta_{3}^{p-1}\right) \tau$ in order to prove the induction step.

To this end, let now $\hat{k} \leq k+1$ be the largest integer such that $t_{0}-t_{\hat{k}} \leq \delta_{3}^{p-1} \tau / 2$. We may without loss of generality assume that $\hat{k}<k+1$, since otherwise $t_{k+1} \geq$ $t-\left(\delta_{1}^{p-1}+\delta_{2}^{p-1}+\delta_{3}^{p-1}\right) \tau$, because $t_{0}>t-\left(\delta_{1}^{p-1}+\delta_{2}^{p-1}\right) \tau$. Now (4.2) and (4.3), together with the fact that $\hat{\delta}_{j}=\delta_{3}$ for $j>\hat{k}$, give

$$
\begin{aligned}
t_{0}-t_{k+1} & =\left(t_{0}-t_{\hat{k}}\right)+\left(t_{\hat{k}}-t_{k+1}\right) \\
& \leq \frac{\delta_{3}^{p-1} \tau}{2}+\sum_{j=\hat{k}}^{k}\left(t_{j}-t_{j+1}\right) \\
& \leq \frac{\delta_{3}^{p-1} \tau}{2}+\left(2^{\hat{k}+1} H\right)^{2-p}\left(r_{\hat{k}} / \sigma\right)^{p}+\sum_{j=\hat{k}+1}^{k}\left(2^{j+1} H\right)^{2-p}\left(r_{j} / \sigma\right)^{p} \\
& \leq \frac{\delta_{3}^{p-1} \tau}{2}+\frac{\delta_{3}^{p-1} \tau}{4}+\frac{\delta_{3}^{p-1} \tau}{4}\left[\sum_{j=0}^{\infty} 2^{-j \delta_{3} p / c_{9}}\right]^{-1} \sum_{j=\hat{k}+1}^{k} 2^{-j \delta_{3} p / c_{9}} \\
& <\delta_{3}^{p-1} \tau .
\end{aligned}
$$


Therefore, since $t_{0}>t-\left(\delta_{1}^{p-1}+\delta_{2}^{p-1}\right) \tau$, we have that

$$
t-t_{k+1}=t-t_{0}+t_{0}-t_{k}<\left(\delta_{1}^{p-1}+\delta_{2}^{p-1}+\delta_{3}^{p-1}\right) \tau
$$

which was to be proven. Hence we have concluded the proof of the induction step. As a consequence, we have constructed a sequence of points $P_{j}=\left(x_{j}, t_{j}\right) \in \widetilde{Q}_{\delta_{1}}$, such that $d\left(x_{j}, \partial \Omega\right) \rightarrow 0$ and $u\left(P_{j}\right) \rightarrow \infty$ as $j \rightarrow \infty$. This violates the assumed continuity of $(u-\lambda)_{+}$in the neighborhood of $S_{T} \cap \overline{\widetilde{Q}_{\delta_{1}}}$, giving the contradiction. Hence,

$$
\sup _{Q} u \leq H+\lambda,
$$

completing the proof of the theorem.

\section{Estimating the boundary type Riesz measure}

In this section we establish, in NTA-cylinders, upper and lower bounds for the measure $\mu$ defined in (1.6).

5.1. Upper estimate on $\mu$. We will first provide an upper bound on the measure. The proof relies on the Carleson estimate in Theorem 4.2 and the following standard Caccioppoli type estimate, see [9].

Lemma 5.1. Let $u$ be a non-negative weak subsolution in $\Omega_{T}$, and $\phi \in C_{0}^{\infty}(\Omega \times$ $\left.\left(t_{1}, T\right)\right)$ with $\phi \geq 0$. Then

$$
\int_{t_{1}}^{t_{2}} \int_{\Omega}|\nabla u|^{p} \phi^{p} \mathrm{~d} x \mathrm{~d} t \leq C\left(\int_{t_{1}}^{t_{2}} \int_{\Omega} u^{p}|\nabla \phi|^{p} \mathrm{~d} x \mathrm{~d} t+\int_{t_{1}}^{t_{2}} \int_{\Omega} u^{2}\left(\phi_{t}\right)_{+} \phi^{p-1} \mathrm{~d} x \mathrm{~d} t\right)
$$

for $C=C(p, n)$.

Theorem 5.2. Let $\Omega \subset \mathbb{R}^{n}$ be an NTA-domain with constants $M$ and $r_{0}$. Let $0<r \leq r_{0}$ and let $u$ be a weak non-negative solution in $\Omega_{T}$. Fix a point $x_{0} \in \partial \Omega$ and define

$$
\tau=\frac{C_{4}}{16}\left[C_{5} u\left(a_{r}\left(x_{0}\right), t_{0}\right)\right]^{2-p} r^{p}
$$

where $C_{4}$ and $C_{5}$, both depending on $p, n$, are as in Theorem 3.9. Let $0<\delta \leq \widetilde{\delta} \leq 1$ and assume that $t_{0}>5 \widetilde{\delta}^{p-1} \tau$ and that $u$ vanishes continuously on $S_{T} \cap\left(B_{r}(x) \times\right.$ $\left.\left(t_{0}-4 \widetilde{\delta}^{p-1} \tau, t_{0}-\delta^{p-1} \tau\right)\right)$ from $\Omega_{T}$. Then there is a constant $C \equiv C(p, n)$ and $c_{8} \equiv$ $c_{8}(p, n, M)$ such that

$$
\frac{\mu(Q)}{r^{n}} \leq C\left(c_{6} / \widetilde{\delta}\right)^{c_{8} / \delta} u\left(a_{r}\left(x_{0}\right), t_{0}\right)
$$

where $\mu$ is the measure from (1.6),

$$
Q:=B_{r / 2}\left(x_{0}\right) \times\left(t_{0}-2 \widetilde{\delta}^{p-1} \tau, t_{0}-\delta^{p-1} \tau\right),
$$

and $c_{6}$ is from Theorem 4.2. Furthermore, constants $C, c_{8}$, are stable as $p \rightarrow 2^{+}$.

Proof. After scaling, we may assume that $u\left(a_{r}\left(x_{0}\right), t_{0}\right)=1$. Let

$$
\hat{Q}=B_{r}\left(x_{0}\right) \times\left(t_{0}-3 \widetilde{\delta}^{p-1} \tau, t_{0}-\delta^{p-1} \tau\right),
$$

and observe, by our assumptions, that Theorem 4.2 implies

$$
\sup _{\hat{Q}} u \leq\left(c_{6} / \widetilde{\delta}\right)^{c_{7} / \delta}=: \Lambda .
$$

As in the construction of the measure $\mu$ in (1.6), we see that extending $u$ to the entire cylinder $\hat{Q}$ as zero, we obtain a weak subsolution in $\hat{Q}$. Take a cut-off function 
$\phi \in C^{\infty}(\hat{Q})$ vanishing on $\partial_{p} \hat{Q}$ such that $0 \leq \phi \leq 1, \phi$ is one on $Q$, and $|\nabla \phi|<4 / r$ and $\left(\phi_{t}\right)_{+}<4 /\left[\widetilde{\delta}^{p-1} \tau\right]$. Then by (1.6), the definition of $\phi$ and Hölder's inequality we get

$$
\begin{aligned}
\int_{\hat{Q}} \phi^{p} \mathrm{~d} \mu & \leq \int_{\hat{Q}}|\nabla u|^{p-1}|\nabla \phi| \phi^{p-1} \mathrm{~d} x \mathrm{~d} t+\int_{\hat{Q}} u\left(\phi_{t}\right)_{+} \phi^{p-1} \mathrm{~d} x \mathrm{~d} t \\
& \leq \frac{4}{r} \int_{\hat{Q}}|\nabla u|^{p-1} \phi^{p-1} \mathrm{~d} x \mathrm{~d} t+\int_{\hat{Q}} u\left(\phi_{t}\right)_{+} \phi^{p-1} \mathrm{~d} x \mathrm{~d} t \\
& \leq \frac{4}{r}|\hat{Q}|^{1 / p}\left(\int_{\hat{Q}}|\nabla u|^{p} \phi^{p} \mathrm{~d} x \mathrm{~d} t\right)^{\frac{p-1}{p}}+\int_{\hat{Q}} u\left(\phi_{t}\right)_{+} \phi^{p-1} \mathrm{~d} x \mathrm{~d} t .
\end{aligned}
$$

Now using Lemma 5.1 and (5.1) we see that

$$
\begin{aligned}
\mu(Q) & \leq C \frac{|\hat{Q}|^{1 / p}}{r}\left(\int_{\hat{Q}} u^{p}|\nabla \phi|^{p}+u^{2}\left(\phi_{t}\right)_{+} \phi^{p-1} \mathrm{~d} x \mathrm{~d} t\right)^{\frac{p-1}{p}}+\int_{\hat{Q}} u\left(\phi_{t}\right)_{+} \phi^{p-1} \mathrm{~d} x \mathrm{~d} t, \\
& \leq C \frac{|\hat{Q}|^{1 / p}}{r}\left(|\hat{Q}|\left(\frac{\Lambda^{p}}{r^{p}}+\frac{\Lambda^{2}}{\widetilde{\delta}^{p-1} \tau}\right)\right)^{\frac{p-1}{p}}+C|\hat{Q}| \frac{\Lambda}{\widetilde{\delta}^{p-1} \tau} \\
& \leq C \frac{|\hat{Q}|}{\widetilde{\delta}^{p-1} r^{p}} \Lambda^{p-1} .
\end{aligned}
$$

After scaling back, this can be rewritten in the homogeneous form

$$
\frac{\mu\left(Q_{r}\right)}{r^{n}} \leq C\left(c_{6} / \widetilde{\delta}\right)^{(p-1) c_{7} / \delta} u\left(a_{r}\left(x_{0}\right), t_{0}\right),
$$

completing the proof with $c_{8}=(p-1) c_{7}$.

5.2. Lower estimate on $\mu$. We next prove the lower bound for the measure $\mu$.

Theorem 5.3. Let $\Omega \subset \mathbb{R}^{n}$ be an NTA-domain with constants $M$ and $r_{0}$, and let $u$ be a weak non-negative solution in $\Omega_{T}$. Fix a point $\left(x_{0}, t_{0}\right) \in \partial \Omega \times(0, T]$, and denote $A_{r}^{-}=\left(a_{r / 2}\left(x_{0}\right), t_{0}\right)$ for $0<r<r_{0}$. There exists $C, \tau_{0}, \tau_{1}$, all depending only on $p, n, M$, such that if

$$
\left(t_{0}-\tau_{0} u\left(A_{r}^{-}\right)^{2-p} r^{p}, t_{0}+\left(\tau_{0}+\tau_{1}\right) u\left(A_{r}^{-}\right)^{2-p} r^{p}\right) \subset(0, T),
$$

and if $u$ vanishes continuously on $S_{T} \cap\left(B_{r}(x) \times\left(t_{0}, t_{0}+\left(\tau_{0}+\tau_{1}\right) u\left(A_{r}^{-}\right)^{2-p} r^{p}\right)\right)$ from $\Omega_{T}$, then

$$
u\left(A_{r}^{-}\right) \leq C \frac{\mu(Q)}{r^{n}},
$$

where $\mu$ is the measure from (1.6) and

$$
Q:=B_{r}\left(x_{0}\right) \times\left(t_{0}+\tau_{0} u\left(A_{r}^{-}\right)^{2-p} r^{p}, t_{0}+\left(\tau_{0}+\tau_{1}\right) u\left(A_{r}^{-}\right)^{2-p} r^{p}\right) .
$$

Furthermore, constants $C, \tau_{0}, \tau_{1}$, are stable as $p \rightarrow 2^{+}$.

To prove Theorem 5.3 we first consider the model problem in Lemma 5.4, and we prove that the measure associated to this model problem is bounded from below by a constant. Returning to Theorem 5.3, we then apply the intrinsic Harnack inequality to obtain a lower bound on the function such that by the comparison principle the solution $v$ in Lemma 5.4 is below our solution $u$. The result then follows by the fact that the corresponding measures are ordered according to Lemma 5.5, a fact easily realized if the domain is smooth, as in this case the measure is just the modulus of the gradient to the power $p-1$.

Lemma 5.4. Let $\Omega \subset \mathbb{R}^{n}$ be an NTA-domain with constants $M$ and $r_{0}=2$. There exists constants $C, T_{M}$, both depending on $p, n, M$, such that if $v$ is a continuous 
solution to the problem

$$
\begin{cases}v_{t}-\Delta_{p} v=0 & \text { in }\left(\Omega \cap B_{2}(0)\right) \times\left(0, T_{0}\right) \\ v=0 & \text { on } \partial\left(\Omega \cap B_{2}(0)\right) \times\left[0, T_{0}\right) \\ v=\chi_{B_{1 /(4 M)}\left(a_{1}(0)\right)} & \text { on }\left(\Omega \cap B_{2}(0)\right) \times\{0\},\end{cases}
$$

then

$$
\mu_{v}\left(B_{2}(0) \times\left(0, T_{0}\right)\right) \geq 1 / C .
$$

Furthermore, constants $C, T_{M}$, are stable as $p \rightarrow 2^{+}$

Proof. To begin with, extend $v$ as zero to the rest of $Q=B_{2}(0) \times\left(0, T_{0}\right)$, i.e., set $v \equiv 0$ in $\left(B_{2}(0) \backslash \Omega\right) \times\left(0, T_{0}\right)$, and let $\mu_{v}$ be the associated measure as in (1.6). Let then $h$ be the solution to the problem

$$
\begin{cases}h_{t}-\Delta_{p} h=0 & \text { in } Q \\ h=w & \text { on } \partial_{p} Q\end{cases}
$$

We observe that the supremum of $h$ and $v$, which is one, is attained at the bottom of the cylinder. Let us now recall the decay estimate in Lemma 4.1, which implies that for $Q_{r}^{\lambda}\left(0, t_{0}\right):=\left(\Omega \cap B_{r}(0)\right) \times\left(t_{0}-\lambda^{2-p} r^{p}, t_{0}\right)$ we have

$$
\sup _{Q_{\sigma^{j}}^{2^{-j}}\left(0, t_{0}\right)} v \leq 2^{-j}
$$

for $j \in \mathbb{N}$ provided that $t_{0} \in\left[1, T_{0}\right]$ and $T_{0}>1$. On the other hand, Lemma 3.3 gives us that

$$
1=f_{B_{(4 M)^{-1}\left(a_{1}(0)\right)}} h(x, 0) \mathrm{d} x \leq C \inf _{z \in B_{(2 M)^{-1}}(0)} u(z, \tau)
$$

with $\tau$ and $C$ depending on $p, n, M$. We then apply Theorem 3.2 in order to get

$$
1 \leq \hat{C} \inf _{\hat{Q}} h, \quad \hat{Q}:=B_{1 / M}(0) \times\left(T_{0} / 2, T_{0}\right)
$$

by properly choosing $T_{0}$ by means of $\tau$ and $C$ to be larger than 2 . We then choose large enough $j^{\star} \in \mathbb{N}$ so that

$$
2^{-j^{\star}} \leq \frac{1}{2 \hat{C}} \quad \text { and } \quad \sigma^{j} \leq 1 / M .
$$

Then, sliding $t_{0}$ along $\left(1, T_{0}\right]$ in $(5.2)$, we obtain by combining (5.2) and (5.3) that there is $r_{1}=r_{1}(p, n, M)$ such that

$$
\inf _{\widetilde{Q}}(h-v) \geq \frac{1}{2 \hat{C}}=: \epsilon, \quad \widetilde{Q}:=B_{r_{1}}(0) \times\left(T_{0} / 2, T_{0}\right),
$$

where $\epsilon \equiv \epsilon(p, n, M)$.

Let us now define $\phi=\min \{h-v, \epsilon\}$, which is vanishing on $\partial_{p} Q$ and is $\epsilon$ on $\widetilde{Q}$. Then from the weak formulation of $h$ and $v$ we get that

$$
\int_{Q \cap\{t<\tau\}}(h-v)_{t} \phi+\left(|\nabla h|^{p-2} \nabla h-|\nabla v|^{p-2} \nabla v\right) \cdot \nabla \phi \mathrm{d} x \mathrm{~d} t=\int_{Q \cap\{t<\tau\}} \phi \mathrm{d} \mu_{w} .
$$

For the time term, we integrate to obtain

$$
\begin{aligned}
\int_{Q \cap\{t<\tau\}}(h-v)_{t} \phi \mathrm{d} x \mathrm{~d} t & =\int_{Q \cap\{t<\tau\}} \partial_{t}\left(\int_{0}^{h-v} \min (s, \epsilon) \mathrm{d} s\right) \mathrm{d} x \mathrm{~d} t \\
& \geq \frac{1}{2} \int_{B_{2}(0)} \phi^{2}(x, \tau) \mathrm{d} x .
\end{aligned}
$$

We also have the elementary inequality

$$
\left(|\nabla h|^{p-2} \nabla h-|\nabla v|^{p-2} \nabla v\right) \cdot \nabla \phi \geq \frac{1}{C}|\nabla \phi|^{p}
$$


since $p>2$. Hence by combining the last three displays we arrive at

$$
\sup _{0<t<T_{0}} \int_{B_{1}(0)} \phi^{2}(x, t) \mathrm{d} x+\int_{Q}|\nabla \phi|^{p} \mathrm{~d} x \mathrm{~d} t \leq C \epsilon \mu_{v}(Q) .
$$

Using the parabolic Sobolev inequality ([9, Corollary I.3.1]) we obtain that

$$
\begin{aligned}
\epsilon^{p}|\widetilde{Q}| & \leq \int_{Q} \phi^{p} \mathrm{~d} x \mathrm{~d} t \\
& \leq C\left(\sup _{0<t<T_{0}} \int_{B_{2}(0)} \phi^{p}(x, t) \mathrm{d} x+\int_{Q}|\nabla \phi|^{p} \mathrm{~d} x \mathrm{~d} t\right) \\
& \leq C\left(\epsilon^{p-2} \sup _{0<t<T_{0}} \int_{B_{2}(0)} \phi^{2}(x, t) \mathrm{d} x+\int_{Q}|\nabla \phi|^{p} \mathrm{~d} x \mathrm{~d} t\right) .
\end{aligned}
$$

Hence we see that

$$
1 \leq C \mu_{v}(Q)
$$

with a constant $C \equiv C(p, n, M)$ through the dependencies of $\epsilon, r_{1}, T_{0}$.

The next lemma provides a comparison estimate for the measures. If two solutions are ordered, then the corresponding measures will be ordered as well.

Lemma 5.5. Let $\Omega \subset \mathbb{R}^{n}$ be a domain. Let $u$ and $v$ be weak solutions in $(\Omega \cap$ $\left.B_{r}(0)\right) \times(0, T)$ such that $u \geq v \geq 0$ and both vanish continuously on the lateral boundary $\left(\partial \Omega \cap B_{r}(0)\right) \times(0, T)$. Then

$$
\mu_{v} \leq \mu_{u} \quad \text { in } B_{r}(0) \times(0, T)
$$

in the sense of measures.

Proof. To show this consider the test function $\phi=\min \left(1,(u-v-\epsilon)_{+} / \epsilon\right) \psi$, where $\psi$ is non-negative and belongs to $C_{0}^{\infty}(Q)$ with $Q=B_{r}(0) \times(0, T)$. Obviously $\phi$ is supported in $\left(\Omega \cap B_{r}(0)\right) \times(0, T)$, because both $u$ and $v$ vanish continuously on the lateral boundary $\left(\partial \Omega \cap B_{r}(0)\right) \times(0, T)$. Since both $u$ and $v$ are weak solutions, we have, by extending them both by zero in $\left(B_{r}(0) \backslash \Omega\right) \times(0, T)$, that

$$
\int_{Q}(u-v)_{t} \phi \mathrm{d} x \mathrm{~d} t+\int_{Q}\left(|\nabla u|^{p-2} \nabla u-|\nabla v|^{p-2} \nabla v\right) \cdot \nabla \phi \mathrm{d} x \mathrm{~d} t=0 .
$$

Let us first treat the time term. Integrating by parts we get that

$$
\begin{aligned}
\int_{Q}(u-v)_{t} \phi \mathrm{d} x \mathrm{~d} t & =\int_{Q} \partial_{t}\left(\int_{0}^{u-v} \min \left(1,(s-\epsilon)_{+} / \epsilon\right) \mathrm{d} s\right) \psi \mathrm{d} x \mathrm{~d} t \\
& =-\int_{Q}\left(\int_{0}^{u-v} \min \left(1,(s-\epsilon)_{+} / \epsilon\right) \mathrm{d} s\right) \partial_{t} \psi \mathrm{d} x \mathrm{~d} t \\
& \rightarrow-\int_{Q}(u-v) \partial_{t} \psi \mathrm{d} x \mathrm{~d} t,
\end{aligned}
$$

as $\epsilon \rightarrow 0$. To treat the elliptic term, we begin by noting that

$$
\nabla \phi=\nabla \psi \min \left(1,(u-v-\epsilon)_{+} / \epsilon\right)+\frac{1}{\epsilon} \psi \nabla(u-v) \chi_{U_{\epsilon}},
$$

where $U_{\epsilon}:=\{u-v>\epsilon\} \cap\left(\Omega \cap B_{r}(0)\right) \times(0, T)$. The second term in (5.6) will give rise to a positive term in $(5.4)$, hence we discard it and obtain an inequality

$$
\begin{aligned}
\int_{Q}\left(|\nabla u|^{p-2} \nabla u-|\nabla v|^{p-2} \nabla v\right) \cdot \nabla \phi \mathrm{d} x \mathrm{~d} t \\
\quad \geq \int_{Q}\left(|\nabla u|^{p-2} \nabla u-|\nabla v|^{p-2} \nabla v\right) \cdot \nabla \psi \min \left(1,(u-v-\epsilon)_{+} / \epsilon\right) \mathrm{d} x \mathrm{~d} t \\
\quad \rightarrow \int_{Q}\left(|\nabla u|^{p-2} \nabla u-|\nabla v|^{p-2} \nabla v\right) \cdot \nabla \psi \mathrm{d} x \mathrm{~d} t
\end{aligned}
$$


as $\epsilon \rightarrow 0$ by dominated convergence. Combining the convergence in (5.5) and (5.7) with (5.4) we arrive at the inequality

$$
-\int_{Q}(u-v) \partial_{t} \psi \mathrm{d} x \mathrm{~d} t+\int_{Q}\left(|\nabla u|^{p-2} \nabla u-|\nabla v|^{p-2} \nabla v\right) \cdot \nabla \psi \mathrm{d} x \mathrm{~d} t \leq 0
$$

after sending $\epsilon \rightarrow 0$. Since the non-negative function $\psi \in C_{0}^{\infty}(Q)$ was arbitrary, (5.8) finishes the proof after recalling the definitions of $\mu_{u}$ and $\mu_{v}$.

We now have all the technical tools to complete the proof of Theorem 5.3.

Proof of Theorem 5.3. Let $u$ be as in Theorem 5.3 with $A_{r}^{-}:=\left(a_{r / 2}\left(x_{0}\right), t_{0}\right)$. Applying the Harnack estimate in Theorem 3.6 yields for a constant $C=C(p, n, M)$

$$
u\left(A_{r}^{-}\right) \leq \widetilde{C} \inf _{y \in B_{r /(8 M)}\left(a_{r / 2}\left(x_{0}\right)\right)} u\left(y, t_{0}+\tau_{0} u\left(A_{r}^{-}\right)^{2-p} r^{p}\right), \quad \tau_{0}:=\frac{C}{(2 M)^{p}},
$$

since

$$
t_{0}-\tau_{0} u\left(A_{r}^{-}\right)^{2-p} r^{p}>0 .
$$

Consider the scaled solution

$$
\hat{u}(x, t)=\frac{1}{\lambda} u\left(x_{0}+\frac{r}{2} x, t_{0}+\tau_{0} u\left(A_{r}^{-}\right)^{2-p} r^{p}+\lambda^{2-p}\left(\frac{r}{2}\right)^{p} t\right), \quad \lambda:=\frac{u\left(A_{r}^{-}\right)}{\widetilde{C}},
$$

so that

$$
\inf _{y \in B_{1 /(4 M)}\left(a_{1}(0)\right)} \hat{u}(y, 0) \geq 1,
$$

and $\Omega$ is mapped to $\hat{\Omega}$ and $\left(x_{0}, t_{0}+\tau_{0} u\left(A_{r}^{-}\right)^{2-p} r^{p}\right)$ to $(0,0)$. The comparison principle shows that the function $v$ defined in Lemma 5.4 satisfies $v \leq \hat{u}$ in $(\hat{\Omega} \cap$ $\left.B_{2}(0)\right) \times\left(0, T_{0}\right)$ provided that

$$
t_{0}+\tau_{0} u\left(A_{r}^{-}\right)^{2-p} r^{p}+\lambda^{2-p}\left(\frac{r}{2}\right)^{p} T_{0}<T .
$$

Thus we choose $\tau_{1}:=\widetilde{C}^{p-2} 2^{-p} T_{0}$ in the statement. Applying Lemmas 5.4 and 5.5

$$
\mu_{\hat{u}}\left(B_{2}(0) \times\left(0, T_{0}\right)\right) \geq \mu_{v}\left(B_{2}(0) \times\left(0, T_{0}\right)\right) \geq 1 / C .
$$

Scaling back to $u$ gives us the result.

\section{Construction of BARriers}

In this section we construct the barriers that will serve as the starting point for the estimates of the decay-rate of the solutions. The upper barrier in Lemma 6.4 is based on the function constructed in [12, Theorem 4.1]. However the subsolution constructed in Lemma 6.1 seems to be new and allows us to obtain $p$-stable estimates from below on the decay rate.

Lemma 6.1. Let $T=\left(n^{p-1} p\right)^{-1}$ and for $a \in(0,1)$, let

$$
\varrho_{0}:=\min \left\{\frac{a p}{n(p-2)}+1,2\right\}^{\frac{p-1}{p}} .
$$

Then the function $h$,

$$
h(x, t)=g(|x|, t)-g\left(\varrho_{0}, t\right), \quad g(r, t)=\left[1-\frac{p-2}{p^{\frac{p}{p-1}}} \frac{r^{\frac{p}{p-1}}-1}{t^{\frac{1}{p-1}}}\right]_{+}^{\frac{p-1}{p-2}},
$$


is a classical subsolution in $\left(B_{\varrho_{0}}(0) \backslash \overline{B_{1}(0)}\right) \times(0, T)$ satisfying the boundary conditions

$$
\begin{cases}h=0, & \text { on } \partial B_{\varrho_{0}}(0) \times(0, T) \\ h=0, & \text { on }\left(B_{\varrho_{0}}(0) \backslash \overline{B_{1}(0)}\right) \times\{0\} \\ h=1-\left[1-\frac{p-2}{p^{p-1}} \frac{\varrho_{0} \frac{p}{p-1}-1}{t^{\frac{1}{p-1}}}\right]_{+}^{\frac{p-1}{p-2}}, & \text { on } \partial B_{1}(0) \times(0, T) .\end{cases}
$$

Furthermore, $x \mapsto h(x, t)$ is a radially decreasing function satisfying

$$
\inf _{1 \leq|x| \leq \varrho_{0}}|\nabla h(x, T)| \geq n \exp \left(-\frac{n}{p}\right)(1-a)^{\frac{n}{p}},
$$

and $h(x, t) \equiv h_{p}(x, t)$ tends to

$$
\exp \left(-\frac{|x|^{2}-1}{4 t}\right)-\exp \left(-\frac{1}{4 t}\right)
$$

as $p \rightarrow 2^{+}$.

Remark 6.2. Note that the function in Lemma 6.1 is not continuous up to the boundary at the corner $\partial B_{1}(0) \times\{0\}$. However, the limsup as we approach a point on this piece from the inside of $D:=\left(B_{\varrho_{0}}(0) \backslash \overline{B_{1}(0)}\right) \times(0, T)$ is 1 for $h$. This implies, see [20, Lemma 4.4], that if we have a weak supersolution $u$ in $D$, staying above the boundary conditions in (6.1) in liminf sense, and staying above 1 on the corner $\partial B_{1}(0) \times\{0\}$ again in the liminf sense, then $u$ will be above $h$ in $D$.

Proof. Let $h$ and $\varrho_{0}$ be as stated. By construction, the boundary conditions for $h$ are in force. To verify that $h$ is a classical subsolution in $\left(B_{\varrho_{0}}(0) \backslash \overline{B_{1}(0)}\right) \times(0, T)$, we first compute,

$$
\begin{aligned}
\nabla g(|x|, t) & =-\frac{x}{|x|}\left[\frac{1}{p} \frac{|x|}{t} g(|x|, t)\right]^{\frac{1}{p-1}} \\
|\nabla g(|x|, t)|^{p-2} \nabla g(|x|, t) & =-\frac{1}{p} \frac{x}{t} g(|x|, t), \\
-\Delta_{p} g(|x|, t) & =\left[\frac{n}{p t} g(|x|, t)^{\frac{p-2}{p-1}}-p^{-\frac{p}{p-1}} \frac{|x|^{\frac{p}{p-1}}}{t^{\frac{p}{p-1}}}\right] g(|x|, t)^{\frac{1}{p-1}}, \\
\partial_{t} g(r, t) & =p^{-\frac{p}{p-1}} \frac{r^{\frac{p}{p-1}}-1}{t^{\frac{p}{p-1}}} g(r, t)^{\frac{1}{p-1}} .
\end{aligned}
$$

Observing that $\partial_{t} h(x, t) \leq \partial_{t} g(|x|, t)$ it is enough to verify $g_{t}-\Delta_{p} g \geq 0$ in $\left(B_{\varrho_{0}}(0) \backslash\right.$ $\left.\overline{B_{1}(0)}\right) \times(0, T)$ for $g>0$. Assuming $g>0$, we see, since $g(|x|, t) \leq 1$ for $|x|>1$, that

$$
\begin{aligned}
\frac{\left(h_{t}-\Delta_{p} h\right)(x, t)}{g(|x|, t)^{\frac{1}{p-1}}} & \leq\left[p^{-\frac{p}{p-1}} \frac{|x|^{\frac{p}{p-1}}-1}{t^{\frac{p}{p-1}}}+\frac{n}{p t} g(|x|, t)^{\frac{p-2}{p-1}}-p^{-\frac{p}{p-1}} \frac{|x|^{\frac{p}{p-1}}}{t^{\frac{p}{p-1}}}\right] \\
& \leq \frac{1}{p t}\left[n-\left(\frac{1}{p t}\right)^{\frac{1}{p-1}}\right] .
\end{aligned}
$$

Since $0<t<T=\left(n^{p-1} p\right)^{-1}$ we have that $h_{t}-\Delta_{p} h \leq 0$ for $|x|>1$ and $t \in(0, T)$. Note also that our choices of parameters are stable as $p \rightarrow 2$. Next, by yet an other explicit calculation we obtain

$$
\inf _{1 \leq|x| \leq \varrho_{0}}|\nabla h(x, T)| \geq\left(\frac{1}{p T} g\left(\varrho_{0}, T\right)\right)^{\frac{1}{p-1}}=n g\left(\varrho_{0}, T\right)^{\frac{1}{p-1}} .
$$


To complete the proof we need to estimate $g\left(\varrho_{0}, T\right)$ from below. To do this we note that

$$
g\left(\varrho_{0}, T\right)^{\frac{1}{p-1}}=\left[1-\frac{n(p-2)}{p}\left(\varrho_{0}^{\frac{p}{p-1}}-1\right)\right]^{\frac{1}{p-2}},
$$

and we consider two cases. First, if $\varrho_{0}=2^{\frac{p-1}{p}}$, then $a p \geq n(p-2)$ and

$$
g\left(\varrho_{0}, T\right)^{\frac{1}{p-1}}=(1-s)^{b / s}, \quad s=\frac{n(p-2)}{p}, \quad b=\frac{n}{p} .
$$

Furthermore, for $\eta \in[0,1)$ we have

$$
\begin{aligned}
(1-\eta)^{\frac{b}{\eta}} & =\exp \left(-\frac{b}{\eta} \sum_{k=1}^{\infty} \frac{\eta^{k}}{k}\right)=\exp \left(-b \sum_{k=1}^{\infty} \frac{\eta^{k-1}}{k}\right)=\exp \left(-b-b \sum_{k=1}^{\infty} \frac{\eta^{k}}{k+1}\right) \\
& \geq \exp \left(-b-b \sum_{k=1}^{\infty} \frac{\eta^{k}}{k}\right)=e^{-b}(1-\eta)^{b} .
\end{aligned}
$$

Since $a p \geq n(p-2)$ implies $s \leq a<1$, we can apply (6.2) to get

$$
g\left(\varrho_{0}, T\right)^{\frac{1}{p-1}} \geq \exp (-b)(1-s)^{b} \geq \exp (-b)(1-a)^{b} .
$$

Second, if $\varrho_{0}<2^{\frac{p-1}{p}}$, then $a p<n(p-2)$ and using $(6.2)$ we get

$$
g\left(\varrho_{0}, T\right)=(1-a)^{\frac{p-1}{p-2}} \geq(1-a)^{b / a} \geq \exp (-b)(1-a)^{b} .
$$

Collecting the results of the two cases completes the proof of the lemma.

Remark 6.3. Note that we could, as in [32], instead of the function in Lemma 6.1 use the Barenblatt fundamental solution together with the barriers from [6] to establish a version of Lemma 6.1. However, this would result in a construction which is not $p$-stable.

In the next lemma we construct a certain supersolution to be used in the subsequent arguments.

Lemma 6.4. Let $T, H>0$ be given degrees of freedom. Let

$$
k \in\left(0, k_{0}\right], \quad k_{0}:=\min \left\{\frac{p-1}{n}, T^{\frac{1}{p-1}} H^{\frac{p-2}{p-1}}\right\} .
$$

There exists a classical supersolution $\widetilde{h}$ in

$$
N=\{(x, t): 1<|x|<1+k, 0<t<T\},
$$

such that

and such that

$$
\begin{cases}\widetilde{h} \geq 0, & \text { in } \partial B_{1}(0) \times(0, T] \\ \widetilde{h} \geq H & \text { on }\left(\overline{B_{1+k}(0)} \backslash B_{1}(0)\right) \times\{0\} \\ \widetilde{h} \geq H & \text { on } \partial B_{1+k}(0) \times[0, T]\end{cases}
$$

$$
\widetilde{h}(x, T) \leq \frac{H \exp (2)}{k}(|x|-1)
$$

whenever $x \in B_{1+k}(0) \backslash B_{1}(0)$.

Proof. This type of construction was originally carried out in [12, Theorem 4.1] and we here include a proof for completion. Let

$$
v(x, t)=\exp \left(\frac{t-T}{T}-\frac{|x|-1}{k}\right),
$$

and let

$$
\widetilde{h}(x, t)=\widetilde{H}(1-v(x, t)), \quad \widetilde{H}=H \exp (2),
$$


accordingly. Then

$$
\partial_{t} \widetilde{h}(x, t)=-\frac{\widetilde{H}}{T} v(x, t) \quad \text { and } \quad \nabla \widetilde{h}(x, t)=\frac{\widetilde{H} x}{k|x|} v(x, t) .
$$

Observe also that $\widetilde{H} v \geq H$ for all $(x, t) \in N$ and that $\widetilde{h}$ satisfies the boundary conditions. We now show that $\widetilde{h}$ is a classical supersolution in $N$. Indeed, by a straightforward calculation we see that

$$
\begin{aligned}
\widetilde{h}_{t}-\Delta_{p} \widetilde{h} & =-\frac{\widetilde{H}}{T} v+\widetilde{H}^{p-1} v^{p-1}\left((p-1) k^{-p}-k^{1-p} \frac{n-1}{|x|}\right) \\
& \geq \frac{\widetilde{H}}{T} v\left(-1+T H^{p-2} k_{0}^{1-p}+T H^{p-2} k^{1-p}\left((p-1) k_{0}^{-1}-n\right)\right) \\
& \geq 0,
\end{aligned}
$$

whenever $(x, t) \in N$. Finally, since

$$
\sup _{1<|x|<1+k}|\nabla \widetilde{h}(x, T)| \leq \frac{\widetilde{H}}{k} \quad \text { and } \quad \widetilde{h}(x, T)=0 \quad \forall x \in \partial B_{1}(0),
$$

we obtain the upper bound for $\widetilde{h}(x, T)$ as well.

\section{Decay estimates and a Change of VARiables}

In this section we prove a lower bound (Lemma 7.2) and an upper bound (Lemma 7.4) on the decay of solutions. The following lemma, which is a change of variables, will be used in the proof of our decay estimates. The proof of the lemma follows from [22, Lemma 3.5].

Lemma 7.1. Let $u=u(x, t)$ be p-parabolic in $\Omega \times\left(T_{0}, T_{1}\right)$. Let $C>0$ be given and let

$$
\begin{aligned}
& f(\tau)=\frac{1}{C(p-2)}(\exp (C(p-2) \tau)-1), \\
& g(\eta)=(C(p-2) \eta+1)^{\frac{1}{p-2}}
\end{aligned}
$$

for $p>2$, and

$$
f(\tau)=\tau, g(\eta)=\exp (C \eta)
$$

for $p=2$. Let $w(x, \tau)=g(f(\tau)) u(x, f(\tau))$. Then $w(x, \tau)$ is a (weak) solution to the equation,

$$
\partial_{\tau} w=\Delta_{p} w+C w
$$

in $\Omega \times\left(\tau_{0}, \tau_{1}\right)$ where $f\left(\tau_{i}\right)=T_{i}, i \in\{0,1\}$.

7.1. A lower bound on the decay. Using the classical subsolution constructed in Lemma 6.1 and the change of variable outlined in Lemma 7.1 we here prove the following lemma, which describes the optimal decay rate from below after a certain intrinsic waiting time. This lemma will be crucial when proving global $C^{1,1}$ estimates, see Section 8 and when proving the local $C^{1,1}$-estimates, see Section 9 .

Lemma 7.2. Let $0<\varrho \leq r / 4$ and let $g \in L^{2}\left(B_{r}\left(x_{0}\right)\right)$ be a non-negative function satisfying

$$
f_{B_{\varrho}\left(x_{0}\right)} g \mathrm{~d} x \geq \lambda>0 .
$$

Assume that $\hat{h} \in C\left(\left[t_{0}, \infty\right) ; L^{2}\left(B_{r}\left(x_{0}\right)\right)\right)$ is a weak non-negative solution solving the Cauchy problem

$$
\begin{cases}\hat{h}_{t}-\Delta_{p} \hat{h}=0 & \text { in } B_{r}\left(x_{0}\right) \times\left(t_{0}, \infty\right) \\ \hat{h}=g & \text { on } B_{r}\left(x_{0}\right) \times\left\{t_{0}\right\}\end{cases}
$$


Then there exist constants $c_{i} \equiv c_{i}(\varrho / r, n, p), i \in\{1,2\}$, such that

$$
\hat{h}(x, t) \geq \frac{\lambda}{c_{1}}\left(c_{1}(p-2) \frac{t-t_{0}}{\lambda^{2-p} r^{p}}+1\right)^{-\frac{1}{p-2}} \frac{d\left(x, \partial B_{r}\left(x_{0}\right)\right)}{r},
$$

whenever $(x, t) \in B_{r}\left(x_{0}\right) \times\left(t_{0}+c_{2} \lambda^{2-p} r^{p}, \infty\right)$. Furthermore, constants $c_{i}, i \in\{1,2\}$, are stable as $p \rightarrow 2^{+}$.

Proof. After scaling we may assume that $x_{0}=0, t_{0}=0, r=1$, and $\lambda=1$. Let

$$
\varrho_{0}:=\min \left\{\frac{1}{2} \frac{p}{n(p-2)}+1,2\right\}^{\frac{p-1}{p}} .
$$

Applying Lemma 3.3 we find a time $t^{\star} \equiv t^{\star}(n, p, \varrho / r)$ and a constant $c^{\star} \equiv c^{\star}\left(n, p, \frac{\varrho}{r}\right)$ such that

$$
\hat{h}\left(x, t^{\star}\right) \geq 1 / c^{\star} \quad \forall x \in B_{1 / \varrho_{0}}(0) .
$$

Set $\bar{h}(x, t)=c^{\star} \hat{h}\left(x / \varrho_{0}, t^{\star}+t\left[c^{\star}\right]^{p-2} / \varrho_{0}^{p}\right)$ and let $w(x, \tau)=g(f(\tau)) \bar{h}(x, f(\tau))$, where $g$ and $f$ are defined as in Lemma 7.1. Then $w(x, \tau)$ is a non-negative weak solution to the equation

$$
\partial_{\tau} w=\Delta_{p} w+C w
$$

in $B_{\varrho_{0}}(0) \times(0, \infty)$ and $w(x, 0) \geq 1$ for all $x \in B_{1}(0)$. In particular, $w$ is a weak supersolution in $B_{\varrho_{0}}(0) \times(0, \infty)$. Now, Theorem 3.2, [22, Corollary 3.6], Lemma 7.1, and [22, Proposition 3.1] imply that we have, for a new constant $\bar{c} \equiv \bar{c}(n, p)>1$,

$$
w(x, \tau) \geq 1 / \bar{c}, \quad(x, \tau) \in B_{1}(0) \times(0, \infty),
$$

provided we choose $C$ large enough in the definitions of $f$ and $g$ in Lemma 7.1. Consider $\hat{\tau} \geq 0$ arbitrary, let $h$ be the classical subsolution of Lemma 6.1 and let $T$ be as in Lemma 6.1. Then, simply using the intrinsic scaling, the comparison principle, and (7.1) we see that

$$
w(x, \tau) \geq \frac{1}{\bar{c}} h\left(x, \bar{c}^{2-p}(\tau-\hat{\tau})\right)
$$

whenever $(x, \tau) \in\left(B_{\varrho_{0}}(0) \backslash B_{1}(0)\right) \times\left(\hat{\tau}, \hat{\tau}+\bar{c}^{p-2} T\right)$. Since $\hat{\tau} \geq 0$ was arbitrary we get from (7.2) and Lemma 6.1 that there is a $c \equiv c(n, p)$ such that

$$
w(x, \tau) \geq \frac{1}{c} d\left(x, \partial B_{\varrho_{0}}(0)\right), \quad(x, \tau) \in B_{\varrho_{0}}(0) \times\left(\bar{c}^{p-2} T, \infty\right) .
$$

To complete the proof, it suffices to rephrase (7.3) in terms of $\hat{h}(x, t)$.

7.2. An upper bound on the decay. Working with solutions vanishing on the entire lateral boundary, we will make use of the following decay estimates.

Lemma 7.3. Let $u \in C\left([0, T) ; L^{2}(\Omega)\right) \cap L^{p}\left(0, T ; W_{0}^{1, p}(\Omega)\right)$ be a non-negative weak subsolution in $\Omega \times(0, T)$. Then there exists a constant $c=c(n, p,|\Omega|)$ such that

$$
\sup _{\Omega} u(\cdot, t) \leq \frac{c}{t^{n / \sigma}}\left(f_{\Omega} u(x, 0) \mathrm{d} x\right)^{p / \sigma}, \quad \sigma=n(p-2)+p .
$$

The constant $c$ is stable as $p \rightarrow 2^{+}$.

Proof. See [25, Lemma 3.24] and use the $L^{1}$-contractivity (test with the function $\min (1, u / \epsilon))$ and the comparison principle.

In the following lemma we describe the optimal decay of the supremum of a solution vanishing on the entire lateral boundary, it follows from an iterative rescaling and comparison together with the decay estimate in Lemma 7.3. 
Lemma 7.4. Let $u \in C\left([0, T) ; L^{2}(\Omega)\right) \cap L^{p}\left(0, \infty ; W_{0}^{1, p}(\Omega)\right)$ be a non-negative weak subsolution in $\Omega \times(0, \infty)$. Then there exist constants $c_{i} \equiv c_{i}(p, n,|\Omega|), i \in\{1,2\}$, such that the following holds. Let

$$
\bar{\Lambda}:=f_{\Omega} u(x, 0) \mathrm{d} x,
$$

then

$$
\sup _{\Omega} u(\cdot, t) \leq c_{1}\left((p-2) \frac{\bar{\Lambda}^{p-2}}{c_{1}} t+1\right)^{-\frac{1}{p-2}} \bar{\Lambda},
$$

whenever $t>c_{2} \bar{\Lambda}^{2-p}$. The constants $c_{i}, i \in\{1,2\}$, are stable as $p \rightarrow 2^{+}$.

Proof. Let $w=w(x, t)$ solve the Dirichlet problem

$$
\begin{cases}w_{t}-\Delta_{p} w=0 & \text { in } \Omega \times(0, \infty) \\ w=0 & \text { on } \partial \Omega \times[0, \infty)\end{cases}
$$

and assume that

$$
f_{\Omega} w(x, 0) \mathrm{d} x \leq 1
$$

Applying Lemma 7.3 to $w$ we see that

$$
\sup _{\Omega} w(\cdot, t) \leq \frac{c}{t^{n / \sigma}},
$$

for some $c=c(n, p,|\Omega|)$, and for all $t>0$. In particular, there exists $t^{\star}=t^{\star}(n, p,|\Omega|)>$ 0 such that

$$
\sup _{\Omega} w\left(\cdot, t^{\star}\right) \leq \frac{1}{2}
$$

To prove the lemma we will now use (7.6) in an iterative argument. In particular, consider the function

$$
w_{1}(x, t):=\bar{\Lambda}^{-1} u\left(x, \bar{\Lambda}^{2-p} t\right) .
$$

Then $w_{1}$ is a solution to (7.4) satisfying (7.5). Hence we have by (7.6) that

$$
w_{1}\left(x, t^{\star}\right) \leq \frac{1}{2}
$$

which after scaling back becomes

$$
u\left(x, \bar{\Lambda}^{2-p} t^{\star}\right) \leq 2^{-1} \bar{\Lambda} \quad \text { whenever } \quad x \in \Omega .
$$

Next, consider the function

$$
w_{2}(x, t):=\left(2^{-1} \bar{\Lambda}\right)^{-1} u\left(x, \bar{\Lambda}^{2-p} t^{\star}+\left(2^{-1} \bar{\Lambda}\right)^{2-p} t\right),
$$

which again satisfies (7.4) and (7.5). Applying (7.6) to the function $w_{2}$ we deduce, by elementary manipulations, that

$$
\sup _{\Omega} u\left(\cdot,\left(\bar{\Lambda}^{2-p}+\left(2^{-1} \bar{\Lambda}\right)^{2-p}\right) t^{\star}\right) \leq 2^{-2} \bar{\Lambda} .
$$

Proceeding inductively we deduce that

$$
\sup _{\Omega} u\left(\cdot, t_{j}\right) \leq 2^{-j} \bar{\Lambda}
$$

where

$$
t_{j}:=t^{\star} \bar{\Lambda}^{2-p} \sum_{k=0}^{j-1} 2^{k(p-2)}, \quad j \in\{1,2, \ldots\} .
$$

To complete the argument consider $t \in\left(t_{1}, \infty\right)$ and let $j$ be the largest $j$ such that $t_{j} \leq t$. Then, by the comparison principle and by construction,

$$
\sup _{x \in \Omega} u(\cdot, t) \leq 2^{-j} \bar{\Lambda}
$$


and

Since

$$
t_{j} \leq t<t_{j+1}
$$

$$
2^{-j}=\left(\frac{\left(2^{p-2}-1\right)}{t^{\star}} \bar{\Lambda}^{p-2} t_{j}+1\right)^{-\frac{1}{p-2}},
$$

and $2^{p-2}-1 \geq \log (2)(p-2)$, by retracing the argument we derive the conclusion of the lemma. Furthermore, the constants $c_{i}, i \in\{1,2\}$, are stable as $p \rightarrow 2^{+}$. In particular, we see that

$$
\left((p-2) c_{1}^{-1}(n, p,|\Omega|) \bar{\Lambda}^{p-2} t+1\right)^{-\frac{1}{p-2}} \rightarrow \exp \left(-c_{1}^{-1}(n, 2,|\Omega|) t\right) .
$$

This completes the proof of the lemma.

\section{Global estimates in $C^{1,1}$-DOmains}

In this section we combine the optimal decay estimate established in Lemma 8.1 together with the barrier function in Lemma 6.4, to obtain the sharp decay estimate from above. Note that taking the initial data to be $+\infty$ allows us to see that this is sharp with respect to the so called "friendly giant", see for example [23].

Lemma 8.1. Let $\Omega \subset \mathbb{R}^{n}$ be a bounded $C^{1,1}$-domain satisfying the ball condition with radius $r_{0}$. Let $u \in C\left([0, \infty) ; L^{2}(\Omega)\right) \cap L^{p}\left(0, \infty ; W_{0}^{1, p}(\Omega)\right)$ be a non-negative p-parabolic function in $\Omega \times(0, \infty)$. Let

$$
\bar{\Lambda}:=f_{\Omega} u(x, 0) \mathrm{d} x .
$$

Then there exists $C_{i} \equiv C_{i}\left(\operatorname{diam} \Omega / r_{0}, n, p\right), i \in\{1,2\}$, such that

$$
u(x, t) \leq C_{1} \bar{\Lambda}\left((p-2) C_{1}^{-1} \bar{\Lambda}^{p-2} t+1\right)^{-\frac{1}{p-2}} d(x, \partial \Omega)
$$

whenever $t>C_{2} \bar{\Lambda}^{2-p}$. Furthermore, constants $C_{i}, i \in\{1,2\}$, are stable as $p \rightarrow 2^{+}$.

Proof. By scaling we can without loss of generality assume that $\bar{\Lambda}=1$. Let $\hat{x}_{0} \in \partial \Omega$ be an arbitrary point. Assume, for simplicity that $a_{2 r_{0}}^{-}\left(\hat{x}_{0}\right)=0$, where $a_{2 r_{0}}^{-}\left(\hat{x}_{0}\right)$ is the exterior corkscrew point as in Remark 2.3. Consider an arbitrary number $\hat{t}$, such that $\hat{t}>c_{2}$ (where $c_{2}$ is from Lemma 7.4). Then, using Lemma 7.4 we see that

$$
u(x, t) \leq c_{1}\left(\frac{p-2}{c_{1}} \hat{t}+1\right)^{\frac{1}{2-p}}=: \bar{\Lambda}_{u}(\hat{t}), \quad x \in \Omega, t \geq \hat{t} .
$$

Construct the function $\widetilde{h}$ in Lemma 6.4 with the choices $\left(T=\bar{\Lambda}_{u}^{2-p}, H=\bar{\Lambda}_{u}\right)$, then $k_{0}$ from Lemma 6.4 simplifies to

$$
k_{0}=\min \left\{\bar{\Lambda}_{u}^{\frac{2-p}{p-1}} \bar{\Lambda}_{u}^{\frac{p-2}{p-1}}, \frac{p-1}{n}\right\}=\min \left\{1, \frac{p-1}{n}\right\} .
$$

Consider now the function $\hat{h}$ defined as follows

$$
\hat{h}(x, t)=\widetilde{h}\left(\frac{x}{r_{0}}, \frac{t-\hat{t}}{r_{0}^{p}}\right) .
$$

The function $\hat{h}$ is a supersolution in

$$
N:=\left(B_{\left(1+1 / k_{0}\right) r_{0}} \backslash \bar{B}_{r_{0}}\right) \times\left(\hat{t}, \hat{t}+\bar{\Lambda}_{u}^{2-p} r_{0}^{p}\right) .
$$

Thus, the comparison principle, Definition 2.2, (8.1) and (8.2) imply that

$$
u(x, t) \leq \hat{h}(x, t) \text { in } N \cap \Omega_{\infty} .
$$

Next, using the upper estimate (6.3) from Lemma 6.4 we see that

$$
u\left(x, \hat{t}+\bar{\Lambda}_{u}^{2-p} r_{0}^{p}\right) \leq C \bar{\Lambda}_{u}\left(\left|\frac{x}{r_{0}}\right|-1\right),
$$


for a constant $C=C(n, p)$. As $\hat{x}_{0} \in \partial \Omega$ is arbitrary, we see, using from (8.1) and (8.3), that

$$
u\left(x, \hat{t}+\Lambda_{u}^{2-p} r_{0}^{p}\right) \leq C d(x, \partial \Omega),
$$

for a new constant $C \equiv C\left(\operatorname{diam} \Omega / r_{0}, n, p\right)$. Furthermore, as $\hat{t}>c_{2}$ was arbitrary, we see that if $t>\bar{\Lambda}_{u}\left(c_{2}\right)^{2-p} r_{0}^{p}+c_{2}:=C_{2}$, then

$$
u(x, t) \leq C_{1}\left((p-2) C_{1}^{-1} t+1\right)^{-\frac{1}{p-2}} d(x, \partial \Omega),
$$

for a constant $C_{1} \equiv C_{1}\left(\operatorname{diam} \Omega / r_{0}, n, p\right)>1$. This completes the proof of the lemma.

The next lemma provides the corresponding lower bound.

Lemma 8.2. Let $\Omega \subset \mathbb{R}^{n}$ be a bounded connected $C^{1,1}$-domain satisfying the ball condition with radius $r_{0}$. Let $u \in C\left([0, \infty) ; L^{2}(\Omega)\right)$ be a non-negative p-parabolic function in $\Omega \times(0, \infty)$. Suppose that there is a ball $B_{4 r_{1}}\left(x_{1}\right) \subset \Omega, r_{1} \in\left(0, r_{0}\right)$, such that

$$
\bar{\Lambda}:=f_{B_{r_{1}}\left(x_{1}\right)} u(x, 0) \mathrm{d} x>0 .
$$

Then there exist $C_{i} \equiv C_{i}\left(\frac{\operatorname{diam} \Omega}{r_{0}}, \frac{r_{1}}{r_{0}}, n, p\right), i \in\{3,4\}$, such that

$$
u(x, t) \geq \frac{\bar{\Lambda}}{C_{3}}\left((p-2) C_{3} \bar{\Lambda}^{p-2} t+1\right)^{-\frac{1}{p-2}} d(x, \partial \Omega),
$$

whenever $x \in \Omega$ and $t>C_{4} \bar{\Lambda}^{2-p}$. Furthermore, constants $C_{i}, i \in\{3,4\}$, are stable as $p \rightarrow 2^{+}$.

Proof. After scaling and translating we may assume that $\bar{\Lambda}=1, x_{1}=0$ and $r_{0}=1$. Note that with these assumptions we have

$$
f_{B_{r_{1}}(0)} u(x, 0) \mathrm{d} x=1 .
$$

Denote now the set

$$
\Omega^{\delta}:=\{x \in \Omega: d(x, \Omega)>\delta\} .
$$

Since $\Omega$ is connected and satisfies the ball condition with radius 1 , we also obtain that $\Omega^{\delta}$ is connected for $\delta \in(0,1 / 2]$ and thus any two points in $\Omega^{\delta}$ can be connected by a Harnack chain of balls of size $\delta / 4$ and with length depending only on $n, p, \operatorname{diam} \Omega$, and $\delta$. By using Lemma 3.3 and (8.4) we then find positive constant $c^{\star}$ and $t^{\star}$, both depending only on $n, p, \operatorname{diam} \Omega$, and $r_{1}$, such that

$$
\inf _{x \in \Omega^{1 / 2}} u\left(x, t^{\star}\right) \geq 1 / c^{\star} .
$$

Lemma 7.2 then proves the result whenever $x \in \Omega^{1}$. Next, let $y \in \Omega \backslash \Omega^{1}$ and let $y^{\star} \in \partial \Omega$ be such that $d(y, \partial \Omega)=\left|y-y^{\star}\right|$. Since $d(y, \partial \Omega) \leq 1$ and since the direction is unique (see Remark 2.3) we have that $y=a_{2 d(y, \partial \Omega)}\left(y^{\star}\right)$. With this at hand we can consider the point $a_{2}\left(y^{\star}\right)$ (which is collinear with $\left.y, y^{\star}\right)$ satisfying

$$
f_{B_{1 / 4}\left(a_{2}\left(y^{\star}\right)\right)} u\left(x, t^{\star}\right) \mathrm{d} x \geq 1 / c^{\star} .
$$

Applying Lemma 7.2 we see that

$$
u(x, t) \geq \frac{1}{c^{\star} c_{1}}\left(c_{1}(p-2)\left[c^{\star}\right]^{p-2}\left(t-t^{\star}\right)+1\right)^{-\frac{1}{p-2}} d\left(x, \partial B_{1}\left(a_{2}\left(y^{\star}\right)\right)\right),
$$

whenever $x \in B_{1}\left(a_{2}\left(y^{\star}\right)\right), t>t^{\star}$. Applying this for $x=y$ completes the proof.

Remark 8.3. Note that our tools are too rough to obtain the lower bound in Lemma 8.2 independent on the distribution of the initial data. To remedy this, we assume that the initial data is positive in a region away from the boundary. 
In the next theorem we combine the results of Lemmas 8.1 and 8.2 to obtain an elliptic type global Harnack estimate.

Theorem 8.4. Let $\Omega \subset \mathbb{R}^{n}$ be a bounded $C^{1,1}$-domain satisfying the ball condition with radius $r_{0}$. Let $u \in C\left([0, \infty) ; L^{2}(\Omega)\right) \cap L^{p}\left(0, \infty ; W_{0}^{1, p}(\Omega)\right)$ be a non-negative p-parabolic function in $\Omega \times(0, \infty)$. Let

$$
\bar{\Lambda}:=f_{\Omega} u(x, 0) \mathrm{d} x .
$$

Assume that $\operatorname{supp} u(\cdot, 0) \subset \Omega^{\delta}=\{x \in \Omega: d(x, \partial \Omega)>\delta\}$. Then there are constants $c_{i} \equiv c_{i}\left(\frac{\operatorname{diam} \Omega}{r_{0}}, \frac{\delta}{r_{0}}, n, p\right), i \in\{1,2\}$, such that

$$
c_{1}^{-1} \leq \frac{u\left(x, t+\epsilon \bar{\Lambda}^{2-p}\right)}{u(x, t)} \leq c_{1},
$$

whenever $\epsilon \in[0,1], x \in \Omega$ and $t \geq c_{2} \bar{\Lambda}^{2-p}$. Furthermore, constants $c_{i}, i \in\{1,2\}$, are stable as $p \rightarrow 2^{+}$.

Proof. In the following we will use the constants $C_{i}, i \in\{1, \ldots, 4\}$, introduced in Lemma 8.1 and Lemma 8.2. Let $t_{0}=\max \left\{C_{2}, C_{4}\right\} \bar{\Lambda}^{2-p}$ and consider $t \geq t_{0}$ and $\epsilon \in(0,1)$. Then, using Lemma 8.1 and Lemma 8.2 we see that

$$
\begin{aligned}
& \frac{u\left(x, t+\epsilon \bar{\Lambda}^{2-p}\right)}{u(x, t)} \leq C_{1} C_{3}\left(\frac{(p-2) C_{1}^{-1}\left(t \bar{\Lambda}^{p-2}+\epsilon\right)+1}{(p-2) C_{2} t \bar{\Lambda}^{p-2}+1}\right)^{-\frac{1}{p-2}}, \\
& \frac{u\left(x, t+\epsilon \bar{\Lambda}^{2-p}\right)}{u(x, t)} \geq \frac{1}{C_{1} C_{3}}\left(\frac{(p-2) C_{3}\left(t \bar{\Lambda}^{p-2}+\epsilon\right)+1}{(p-2) C_{1}^{-1} t \bar{\Lambda}^{p-2}+1}\right)^{-\frac{1}{p-2}} .
\end{aligned}
$$

Theorem 8.5 follows from this by elementary manipulations. We omit further details.

In the next theorem we use Lemmas 8.1 and 8.2 together with $C^{1, \alpha}$ estimates for weak solution to obtain a global boundary Harnack principle as well as Hölder continuity of ratios of solutions. The intrinsic time interval ensures that the estimate is $p$-stable.

Theorem 8.5. Let $\Omega \subset \mathbb{R}^{n}$ be a bounded $C^{1,1}$-domain satisfying the ball condition with radius $r_{0}$. Let $u, v \in C\left([0, \infty) ; L^{2}(\Omega)\right) \cap L^{p}\left(0, \infty ; W_{0}^{1, p}(\Omega)\right)$ be non-negative p-parabolic functions in $\Omega \times(0, \infty)$. Let

$$
\bar{\Lambda}_{u}=f_{\Omega} u(x, 0) \mathrm{d} x, \quad \bar{\Lambda}_{v}=f_{\Omega} v(x, 0) \mathrm{d} x .
$$

Assume that the initial data is distributed as follows

$$
\operatorname{supp} u(\cdot, 0), \operatorname{supp} v(\cdot, 0) \subset \Omega^{\delta}=\{x \in \Omega: d(x, \partial \Omega)>\delta\} .
$$

Then there exists $\bar{C}_{1} \equiv \bar{C}_{1}\left(\frac{\operatorname{diam} \Omega}{r_{0}}, \frac{\delta}{r_{0}}, n, p\right)$, such that if $\bar{C}_{1} \leq T_{-} \leq T_{+}$satisfy

$$
T_{-} \min \left\{\bar{\Lambda}_{u}, \bar{\Lambda}_{v}\right\}^{2-p} \leq T_{+} \max \left\{\bar{\Lambda}_{u}, \bar{\Lambda}_{v}\right\}^{2-p},
$$

the following holds. There exist $\bar{C}_{i} \equiv \bar{C}_{i}\left(\frac{\operatorname{diam} \Omega}{r_{0}}, \frac{\delta}{r_{0}}, T_{-}, T_{+}, n, p\right), i \in\{2,3\}$, such that

$$
\bar{C}_{2}^{-1} \frac{\bar{\Lambda}_{u}}{\bar{\Lambda}_{v}} \leq \frac{u(x, t)}{v(x, t)} \leq \bar{C}_{2} \frac{\bar{\Lambda}_{u}}{\bar{\Lambda}_{v}}
$$

whenever

$$
(x, t) \in D:=\Omega \times\left(T_{-} \min \left\{\bar{\Lambda}_{u}, \bar{\Lambda}_{v}\right\}^{2-p}, T_{+} \max \left\{\bar{\Lambda}_{u}, \bar{\Lambda}_{v}\right\}^{2-p}\right) .
$$

Furthermore, there exists an exponent $\sigma \equiv \sigma(n, p) \in(0,1)$, such that

$$
\left|\frac{u(x, t)}{v(x, t)}-\frac{u(y, s)}{v(y, s)}\right| \leq \bar{C}_{3} \frac{\bar{\Lambda}_{u}}{\bar{\Lambda}_{v}}\left(|x-y|+\max \left\{\bar{\Lambda}_{u}, \bar{\Lambda}_{v}\right\}^{\frac{2-p}{p}}|t-s|^{1 / p}\right)^{\sigma}
$$


whenever $(x, t),(y, s) \in D$. The constants $\bar{C}_{i}, i \in\{1,2,3\}$, and $\sigma$ are stable as $p \rightarrow 2^{+}$.

Proof. In the following we will let $C_{1}, C_{2}, C_{3}$ and $C_{4}$ be the constants in Lemmas 8.1 and 8.2. We begin by proving (8.6). Indeed, using Lemmas 8.1 and 8.2 we see that

$$
\frac{\bar{\Lambda}_{u}}{\bar{\Lambda}_{v}} \frac{1}{C_{1} C_{3}}\left(\frac{(p-2) C_{1}^{-1} \bar{\Lambda}_{v}^{p-2} t+1}{(p-2) C_{3} \bar{\Lambda}_{u}^{p-2} t+1}\right)^{\frac{1}{p-2}} \leq \frac{u(x, t)}{v(x, t)},
$$

and

$$
\frac{u(x, t)}{v(x, t)} \leq \frac{\bar{\Lambda}_{u}}{\bar{\Lambda}_{v}} C_{1} C_{3}\left(\frac{(p-2) C_{3} \bar{\Lambda}_{v}^{p-2} t+1}{(p-2) C_{1}^{-1} \bar{\Lambda}_{u}^{p-2} t+1}\right)^{\frac{1}{p-2}},
$$

whenever $t \geq \max \left\{C_{2}, C_{4}\right\} \min \left\{\bar{\Lambda}_{u}, \bar{\Lambda}_{v}\right\}^{2-p}$. In particular we see that if $T_{-}>$ $\max \left\{C_{2}, C_{4}\right\}$, and if $T_{-}, T_{+}$satisfy (8.5), then (8.6) holds with a constant $\bar{C}_{2}$ depending only on $\frac{\operatorname{diam} \Omega}{r_{0}}, \frac{\delta}{r_{0}}, T_{-}, T_{+}, n, p$.

To proceed, consider the rescaled $p$-parabolic functions

$$
\widetilde{u}(x, t)=\frac{u\left(x, \bar{\Lambda}_{u}^{2-p} t\right)}{\bar{\Lambda}_{u}}, \quad \widetilde{v}(x, t)=\frac{v\left(x, \bar{\Lambda}_{v}^{2-p} t\right)}{\bar{\Lambda}_{v}} .
$$

Using Lemma 7.4 for $w \in\{\widetilde{u}, \widetilde{v}\}$ we get for $t>c_{2}$ that

$$
\sup _{x \in \Omega} w(x, t) \leq c_{1}\left((p-2) c_{1}^{-1} t+1\right)^{-\frac{1}{p-2}} \leq c_{1},
$$

where $c_{1}, c_{2}$ are as in Lemma 7.4. Thus we can apply [28, Theorem 0.1] to conclude that there exist $C$ and $\sigma$, depending on $\Omega, p$ and $n$, such that, for $w \in\{\widetilde{u}, \widetilde{v}\}$,

$$
|\nabla w(x, t)-\nabla w(\widetilde{x}, \widetilde{t})| \leq C\left(|x-\widetilde{x}|+|t-\widetilde{t}|^{1 / p}\right)^{\sigma},
$$

whenever $(x, t),(\widetilde{x}, \widetilde{t}) \in \Omega \times\left(c_{2}, \infty\right)$. In particular, arguing as in [24, (3.31), p. 2717], we have, for $w \in\{\widetilde{u}, \widetilde{v}\}$,

$$
\left|\frac{w(x, t)}{d(x, \partial \Omega)}-\frac{w(\widetilde{x}, \widetilde{t})}{d(\widetilde{x}, \partial \Omega)}\right| \leq C\left(|x-\widetilde{x}|+|t-\widetilde{t}|^{1 / p}\right)^{\sigma},
$$

whenever $(x, t),(\widetilde{x}, \widetilde{t}) \in \Omega \times\left(c_{2}, \infty\right)$. Scaling back to the original $u, v,(8.10)$ becomes, with $w \in\{u, v\}$,

$$
\left|\frac{w(x, t)}{d(x, \partial \Omega)}-\frac{w(\widetilde{x}, \widetilde{t})}{d(\widetilde{x}, \partial \Omega)}\right| \leq \bar{\Lambda}_{w} C\left(|x-\widetilde{x}|+\bar{\Lambda}_{w}^{\frac{p-2}{p}}|t-\widetilde{t}|^{1 / p}\right)^{\sigma},
$$

whenever $(x, t),(\widetilde{x}, \widetilde{t}) \in \Omega \times\left(c_{2} \bar{\Lambda}_{w}^{p-2}, \infty\right)$. Next, using the identity

$$
\begin{aligned}
\frac{u(x, t)}{v(x, t)}-\frac{u(y, s)}{v(y, s)}= & \frac{d(x, \partial \Omega)}{v(x, t)}\left(\frac{u(x, t)}{d(x, \partial \Omega)}-\frac{u(y, s)}{d(y, \partial \Omega)}\right) \\
& +\frac{u(y, s)}{v(y, s)} \frac{d(x, \partial \Omega)}{v(x, t)}\left(\frac{v(y, s)}{d(y, \partial \Omega)}-\frac{v(x, t)}{d(x, \partial \Omega)}\right),
\end{aligned}
$$

assuming that $s, t \in\left(T_{-} \min \left\{\bar{\Lambda}_{u}, \bar{\Lambda}_{v}\right\}^{2-p}, T_{+} \max \left\{\bar{\Lambda}_{u}, \bar{\Lambda}_{v}\right\}^{2-p}\right)$ and that $T_{-}>c_{2}$ (where $c_{2}$ is from Lemma 7.4), we can apply Lemmas 8.1 and 8.2 together with (8.6) and (8.11) in the identity (8.12) to obtain (8.7). This completes the proof of Theorem 8.5 .

Remark 8.6. Considering the estimates (8.8) and (8.9) in the proof of Theorem 8.5, we see that the nonlinearities dominate for large values of $t$. In particular, there exists a $p$-instable constant $C \equiv C\left(\frac{\operatorname{diam} \Omega}{r_{0}}, \frac{\delta}{r_{0}}, n, p\right)$ such that

$$
C^{-1} \leq \lim _{t \rightarrow \infty} \frac{u(x, t)}{v(x, t)} \leq C,
$$


whenever $x \in \Omega$. Note that $C$ is independent of the initial data. This does not happen when $p=2$ and the effect is purely non-linear.

Remark 8.7. Note that to prove Theorem 8.5 we rely on estimates established in Lemmas 8.1 and 8.2, instead of relying on the comparison principle, the Carleson estimate and the Harnack inequality as in [14, Theorem 2.1]. This is why our estimates from below depend on the distribution of the initial data. Furthermore, this falls fairly short of the result in [14], but none the less we provide a $p$-stable version of the phenomena involved in our case.

\section{LOCAL ESTIMATES IN $C^{1,1}$-DOMAINS}

In this section the main focus is to develop an intrinsic version of the boundary Harnack principle (1.2), see Section 9.2. To do this, we first prove an upper and a lower decay rate estimate in the next section.

9.1. Upper and lower bound on the decay. We begin with the upper bound, which follows by combining the barrier function from Lemma 6.4 together with the Carleson estimate Theorem 4.2. In the following $M$ will denote the NTA-constant of the $C^{1,1}$ domain $\Omega$, see Remark 2.3.

Lemma 9.1. Let $u$ be a non-negative solution in $\Omega_{T}$, where $\Omega$ is a $C^{1,1}$ domain satisfying the ball condition with radius $r_{0}$. Let $x_{0} \in \partial \Omega$ and $0<r \leq r_{0}$. Let $0<\delta \leq \widetilde{\delta} \leq 1$. Assume that $u\left(a_{r}\left(x_{0}\right), t_{0}\right)>0$ for a fixed $t_{0} \in(0, T)$ and let

$$
\tau=\frac{C_{4}}{16}\left[C_{5} u\left(a_{r}\left(x_{0}\right), t_{0}\right)\right]^{2-p} r^{p}
$$

where $C_{4}$ and $C_{5}$, both depending on $p, n$, are as in Theorem 3.9 and with $t_{0}>$ $5 \widetilde{\delta}^{p-1} \tau$. Assume furthermore that $u$ vanishes continuously on $S_{T} \cap\left(B_{r}\left(x_{0}\right) \times\left(t_{0}-\right.\right.$ $\left.\left.4 \widetilde{\delta}^{p-1} \tau, t_{0}-\delta^{p-1} \tau\right)\right)$. Then there exist constants $c_{i} \equiv c_{i}(M, p, n), i \in\{8,9\}$, such that

$$
\sup _{Q} u \leq\left(c_{8} / \widetilde{\delta}\right)^{c_{9} / \delta} \frac{d\left(x_{0}, \partial \Omega\right)}{r} u\left(a_{r}\left(x_{0}\right), t\right),
$$

where $Q:=\left(B_{r}\left(x_{0}\right) \cap \Omega\right) \times\left(t_{0}-2 \widetilde{\delta}^{p-1} \tau, t_{0}-\delta^{p-1} \tau\right)$. Furthermore, constants $c_{i}$, $i \in\{8,9\}$, are stable as $p \rightarrow 2^{+}$

Proof. Without loss of generality, we may after scaling assume that $u\left(a_{r}\left(x_{0}\right), t_{0}\right)=1$ and $r=4$. Applying Lemma 6.4 and Theorem 4.2, with

$$
k:=\min \left\{\frac{p-1}{n}, \widetilde{\delta}\left(\frac{C_{4}}{16} C_{5}^{2-p}\right)^{\frac{1}{p-1}}, 1\right\}, \quad H:=\left(c_{6} / \widetilde{\delta}\right)^{c_{7} / \delta}
$$

with constants as in Theorem 4.2 we get

$$
u(x, t) \leq H
$$

whenever $(x, t) \in\left(B_{4}\left(x_{0}\right) \cap \Omega\right) \times\left(t_{0}-3 \widetilde{\delta}^{p-1} \tau, t_{0}-\delta^{p-1} \tau\right)$. The comparison function indexed by its initial time $s_{0}$ and center point $y_{0}$, is

$$
\hat{h}_{y_{0}, s_{0}}(x, t)=\widetilde{h}\left(x-y_{0}, t-s_{0}\right)
$$

with $T=\widetilde{\delta}^{p-1} \tau$, where $\widetilde{h}$ is from Lemma 6.4. Let $y \in \partial \Omega \cap B_{1}\left(x_{0}\right)$ and consider $y_{0}=a_{2}(y)$, an outer corkscrew point as in Remark 2.3. Then, by the comparison principle

$$
u(x, t) \leq \hat{h}_{y_{0}, s_{0}}(x, t),
$$

in

$$
\left(\Omega \cap\left[B_{1+k}\left(y_{0}\right) \backslash B_{1}\left(y_{0}\right)\right]\right) \times\left(s_{0}, s_{0}+T\right),
$$


whenever

$$
\left(s_{0}, s_{0}+T\right) \subset\left(t_{0}-3 \widetilde{\delta}^{p-1} \tau, t_{0}-\delta^{p-1} \tau\right) .
$$

From Lemma 6.4 and (9.1) we have the estimate

$$
u(x, t) \leq \frac{H \exp (2)}{k}\left(\left|y_{0}-x\right|-1\right),
$$

whenever

$$
(x, t) \in\left(\Omega \cap\left[B_{1+k}\left(y_{0}\right) \backslash B_{1}\left(y_{0}\right)\right]\right) \times\left(t_{0}-2 \widetilde{\delta}^{p-1} \tau, t_{0}-\delta^{p-1} \tau\right),
$$

and $y_{0} \in \partial \Omega \cap B_{1}\left(x_{0}\right)$. From this the result follows by scaling back.

The following lemma establishes to local lower bound on the decay, by combining the barrier from Lemma 6.1 and the Harnack estimates in Theorem 3.6.

Lemma 9.2. Let $u$ be a non-negative solution in $\Omega_{T}$, where $\Omega$ is a $C^{1,1}$ domain satisfying the ball condition with radius $r_{0}$. Let $x_{0} \in \partial \Omega$ and let $0<r<r_{0}$ be fixed. Let $A^{-}=\left(a_{r}\left(x_{0}\right), t_{0}\right), \theta_{-}=u\left(A^{-}\right)^{2-p}$ and $t_{0} \in(0, T)$. There exists constants $c_{i} \equiv c_{i}(M, p, n), i \in\{3,4\}$, such that if

$$
\theta_{-} r^{p}<t_{0}, \quad \text { and } \quad t_{0}+2 c_{4} \theta_{-} r^{p}<T \text {, }
$$

then

$$
\frac{1}{c_{3}} \frac{d(x, \partial \Omega)}{r} u\left(A^{-}\right) \leq u(x, t)
$$

for $x \in B_{r}\left(x_{0}\right) \cap \Omega$ and $t_{0}+c_{4} \theta_{-} r^{p}<t<t_{0}+2 c_{4} \theta_{-} r^{p}$. Furthermore, constants $c_{i}$, $i \in\{3,4\}$, are stable as $p \rightarrow 2^{+}$.

Proof. Set $\lambda_{-}:=u\left(A^{-}\right)$and consider the scaled solution

$$
v(y, s)=\frac{1}{\lambda_{-}} u\left(x_{0}+r y, t_{0}+s \lambda_{-}^{2-p} r^{p}\right) .
$$

Set also $\widetilde{\Omega}:=\left\{y: x_{0}+r y \in \Omega\right\}$ so that $0 \in \partial \widetilde{\Omega}$. For the new function $v$ we have the following situation, denoting $A_{v}^{-}=\left(\left(a_{r}\left(x_{0}\right)-x_{0}\right) / r, 0\right)$,

$$
\begin{aligned}
v\left(A_{v}^{-}\right) & =1, \\
d\left(A^{-}, \partial \widetilde{\Omega}\right) & =1,
\end{aligned}
$$

and $v$ is a solution in $\widetilde{\Omega} \times(-1, \widetilde{T})$, where $\widetilde{T}:=\left(T-t_{0}\right) \lambda_{-}^{p-2} r^{-p}$. Since $0<r<r_{0} / 4$ we know that $\widetilde{\Omega}$ satisfies the ball condition with radius 4 . To continue, consider the following set

$$
D=\left\{y \in \widetilde{\Omega}: d\left(y, B_{1}(0) \cap \partial \widetilde{\Omega}\right)=d(y, \partial \widetilde{\Omega})=1\right\} .
$$

Note that $D \subset B_{2}(0) \cap \widetilde{\Omega}$ and that $\sup \left\{d\left(a_{1}\left(y_{0}\right), y\right): y \in D\right\} \leq 2$ for any $y_{0} \epsilon$ $\partial \widetilde{\Omega} \cap B_{1}(0)$. We obtain from the Harnack chain estimate in Theorem 3.6 (applied with $\delta=\min \left\{c_{h}^{(2-p) / p}, 1\right\}$ where $c_{h}$ is from Theorem 3.6), that there is a $\widetilde{\tau}_{1}>0$ depending only on $n, p, M$ such that

$$
v\left(x, \widetilde{\tau}_{1}\right) \geq \frac{1}{\widetilde{c}_{1}}
$$

whenever $x \in\{y: d(y, D)<1 / 4\}$ provided $\widetilde{T}>\widetilde{\tau}_{1}$. Using Lemma 7.2 (applied with $\left.r=1, \varrho=1 / 4, g=v\left(\cdot, \widetilde{\tau}_{1}\right), x_{0}=\widetilde{y} \in D, t_{0}=\widetilde{\tau}_{1}\right)$ for all points $\widetilde{y} \in D$, we get

$$
v(y, t) \geq \frac{1}{\widetilde{c}_{1} c_{1}}\left(c_{1} \widetilde{c}_{1}^{2-p}(p-2)\left(t-\widetilde{\tau}_{2}\right)+c_{1} c_{2}(p-2)+1\right)^{-\frac{1}{p-2}} d(y, \widetilde{\Omega})
$$

whenever $(y, t) \in\left(\widetilde{\Omega} \cap B_{1}(0)\right) \times\left(\widetilde{\tau_{2}}, \widetilde{T}\right)$, with $\widetilde{\tau}_{2}=\widetilde{\tau}_{1}+c_{2} \widetilde{c}_{1}^{p-2}$ provided $\widetilde{T}>\widetilde{\tau}_{2}$. Going back to $\Omega$ and $u$ gives us the result provided $\widetilde{T}>2 \widetilde{\tau}_{2}$.

Combining Lemmas 9.1 and 9.2 we obtain the joint estimate. 
Theorem 9.3. Let $u$ be a non-negative solution in $\Omega_{T}$, where $\Omega$ is a $C^{1,1}$ domain satisfying the ball condition with radius $r_{0}$. Let $x_{0} \in \partial \Omega, t_{0} \in(0, T)$, and let $0<$ $r<r_{0}$ be fixed. Let $A^{-}=\left(a_{r}\left(x_{0}\right), t_{0}\right)$ and $\theta_{-}=u\left(A^{-}\right)^{2-p}$. There exists constants $c_{i} \equiv c_{i}(M, p, n), i \in\{5,6\}$, such that if

$$
\theta_{-} r^{p}<t_{0}, \quad \text { and } \quad t_{0}+2 c_{4} \theta_{-} r^{p}<T,
$$

then for $A^{+}=\left(a_{r}\left(x_{0}\right), t_{0}+2 c_{4} \theta_{-} r^{p}\right)$ and $\theta_{+}=c_{6}^{-1} u\left(A^{+}\right)^{2-p}$ (where $c_{4}$ is from Lemma 9.2), we have

$$
5 \theta_{+} \leq \theta_{-} .
$$

Furthermore, if $u$ vanishes continuously on

$$
S_{T} \cap\left(B_{r}\left(x_{0}\right) \times\left(t_{0}+\left[2 c_{4} \theta_{-}-5 \theta_{+}\right] r^{p}, t_{0}+\left[2 c_{4} \theta_{-}-\theta_{+}\right] r^{p}\right)\right)
$$

then

$$
\frac{1}{c_{5}} \frac{d(x, \partial \Omega)}{r} u\left(A^{-}\right) \leq u(x, t) \leq c_{5} \frac{d(x, \partial \Omega)}{r} u\left(A^{+}\right)
$$

for $x \in B_{r}\left(x_{0}\right) \cap \Omega$ and $t_{0}+\left[2 c_{4} \theta_{-}-2 \theta_{+}\right] r^{p}<t<t_{0}+\left[2 c_{4} \theta_{-}-\theta_{+}\right] r^{p}$. Furthermore, constants $c_{i}, i \in\{5,6\}$, are stable as $p \rightarrow 2^{+}$.

Proof. Rescale $u$ as in the proof of Lemma 9.2, let also $\widetilde{\tau}_{2}$ be as in the proof of Lemma 9.2. Thus

$$
\frac{1}{c_{3}} d(x, \partial \Omega) \leq v(x, t)
$$

holds for $(x, t)\left(B_{r}\left(x_{0}\right) \cap \Omega\right) \times\left(\widetilde{\tau}_{2}, 2 \widetilde{\tau}_{2}\right)$. Define $\tau_{+}=2 \widetilde{\tau}_{2}$ and consider $A_{+}=\left(a_{1}(0), \tau_{+}\right)$. Then using (9.2) we get

$$
\begin{aligned}
v\left(A_{+}\right)^{2-p} & \leq\left[\frac{1}{\widetilde{c}_{1} c_{1}}\left(c_{1} \widetilde{c}_{1}^{2-p}(p-2)\left(t-\widetilde{\tau}_{2}\right)+c_{1} c_{2}(p-2)+1\right)^{-\frac{1}{p-2}}\right]^{2-p} \\
& =\frac{1}{\left(\widetilde{c}_{1} c_{1}\right)^{2-p}}\left[c_{1} \widetilde{c}_{1}^{2-p}(p-2)+c_{1} c_{2}(p-2)+1\right] \\
& =: \widetilde{c}_{2} .
\end{aligned}
$$

We will now apply Lemma 9.1 with $\left(r=1, \delta^{p-1}=\min \left\{\frac{16}{5 \widetilde{c}_{2} C_{4}} C_{5}^{p-2}, 1\right\}\right.$ and $\widetilde{\delta}=\delta$, with $C_{4}, C_{5}$ from Lemma 9.1). Doing this we see that

$$
v(x, t) \leq \widetilde{c_{3}} d(x, \partial \widetilde{\Omega}) v\left(A_{+}\right),
$$

whenever $(x, t) \in\left(B_{1}(0) \cap \Omega\right) \times\left(\tau_{+}-v\left(A_{+}\right)^{2-p}, \tau_{+}-v\left(A_{+}\right)^{2-p} / 2\right)$. Going back to $\Omega$ and $u$ gives us the result.

9.2. Local boundary Harnack estimate. We are now ready to state and prove our local boundary Harnack principle, consult Figure 1 for a schematic of the geometry.

Theorem 9.4. Let $u, v$ be two non-negative solutions in $\Omega_{T}$, where $\Omega$ is a $C^{1,1}$ domain satisfying the ball condition with radius $r_{0}$. Let $x_{0} \in \partial \Omega t_{0} \in(0, T)$, and $0<r<r_{0}$ be fixed. Let $A_{-}=\left(a_{r}\left(x_{0}\right), t_{0}\right)$ and assume that $u\left(A_{-}\right)=v\left(A_{-}\right)$. Let the constants $c_{i}, i \in\{4,5,6\}$ be as in Lemma 9.2 and Theorem 9.3. Let $\theta_{-}=u\left(A_{-}\right)^{2-p}$, and assume

$$
\theta_{-} r^{p}<t_{0}, \quad \text { and } \quad t_{0}+2 c_{4} \theta_{-} r^{p}<T .
$$

Set

$$
A_{+}=\left(a_{r}\left(x_{0}\right), t_{0}+2 c_{4} \theta_{-} r^{p}\right), \quad \theta_{+, u}=c_{6}^{-1} u\left(A_{+}\right)^{2-p} .
$$

Assume that $v\left(A_{+}\right) \geq u\left(A_{+}\right)$. Then there exists a time $t_{+}^{\star}$, depending on $v$, satisfying

$$
\begin{aligned}
& t_{+}^{\star} \in\left(t_{0}+\left(2 c_{4} \theta_{-}-\theta_{+, u}\right) r^{p}, t_{0}+2 c_{4} \theta_{-} r^{p}\right) \\
& A_{+}^{\star}=\left(a_{r}\left(x_{0}\right), t_{+}^{\star}\right) \\
& \theta_{+, v}^{\star}=c_{6}^{-1} v\left(A_{+}^{\star}\right)^{2-p},
\end{aligned}
$$


such that the following holds. If both $u$ and $v$ vanish continuously on

$$
S_{T} \cap\left(B_{r}\left(x_{0}\right) \times\left(t_{0}+\left[2 c_{4} \theta_{-}-5 \theta_{+, u}\right] r^{p}, t_{0}+\left[2 c_{4} \theta_{-}-\theta_{+, u}\right] r^{p}\right)\right),
$$

then

$$
\frac{1}{c_{5}^{2}} \frac{u\left(A_{-}\right)}{v\left(A_{+}^{\star}\right)} \leq \frac{u(x, t)}{v(x, t)} \leq c_{5}^{2} \frac{u\left(A_{+}\right)}{v\left(A_{-}\right)},
$$

whenever $(x, t)$ belongs to the set

$$
\left(B_{r}\left(x_{0}\right) \cap \Omega\right) \times\left(t_{0}+\left[2 c_{4} \theta_{-}-\left(\theta_{+, v}^{\star}+\theta_{+, u}\right)\right] r^{p}, t_{0}+\left[2 c_{4} \theta_{-}-\theta_{+, u}\right] r^{p}\right) .
$$

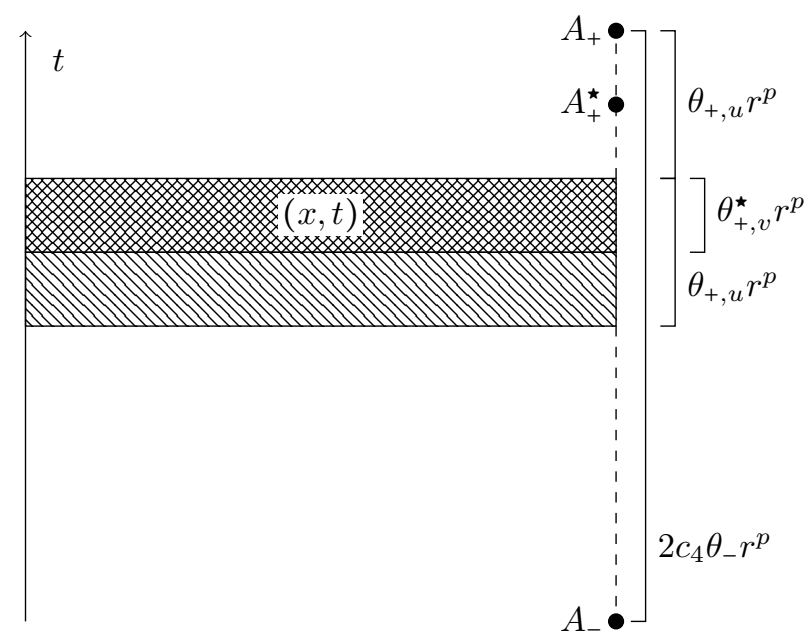

FiguRE 1. The boxes ( $)$ denotes the region where the righthand-side/left-hand-side of Theorem 9.4 holds respectively.

Remark 9.5. It should be noted that we cannot control the time $t_{+}^{\star}$ except which interval it lies in, it is a purely intrinsic parameter. Furthermore note that Theorem 9.4 is equivalent to the boundary Harnack principle (1.2) when $p=2$.

Proof. Let $c_{i}, i \in\{3, \ldots, 6\}$ be as in Lemma 9.2 and Theorem 9.3. By the assumptions, we know that $\theta_{+, u} \geq \theta_{+, v}:=c_{6}^{-1} v\left(A_{+}\right)^{2-p}$. We then obtain by Theorem 9.3, for $x \in B_{r}\left(x_{0}\right) \cap \Omega$ and $t_{0}+\left(2 c_{4} \theta_{-}-2 \theta_{+, u}\right) r^{p}<t<t_{0}+\left(2 c_{4} \theta_{-}-\theta_{+, u}\right) r^{p}$, that

$$
\frac{1}{c_{5}} \frac{d(x, \partial \Omega)}{r} u\left(A_{-}\right) \leq u(x, t) \leq c_{5} \frac{d(x, \partial \Omega)}{r} u\left(A_{+}\right) .
$$

Using Lemma 9.2 for $x \in B_{r}\left(x_{0}\right) \cap \Omega$ and $t_{0}+c_{4} \theta_{-} r^{p}<t<t_{0}+2 c_{4} \theta_{-} r^{p}$ we get that

$$
\frac{1}{c_{3}} \frac{d(x, \partial \Omega)}{r} v\left(A_{-}\right) \leq v(x, t) \text {. }
$$

Now let $t_{+}=t_{0}+2 c_{4} \theta_{-} r^{p}$, and let $t_{+}^{\star}$, a time to be fixed, such that $t_{+}-\theta_{+, u} r^{p}<t_{+}^{\star} \leq t_{+}$. First note that if $t_{+}^{\star}=t_{+}$we have for $\theta_{+, v}^{\star}=c_{6}^{-1}\left(v\left(a_{r}\left(x_{0}\right), t_{+}^{\star}\right)\right)^{2-p}$,

$$
t_{+}^{\star}-\theta_{+, v}^{\star} r^{p} \geq t_{+}-\theta_{+, u} r^{p}
$$

furthermore if $t_{+}^{\star}=t_{+}-\theta_{+}^{u} r^{p}$ then

$$
t_{+}^{\star}-\theta_{+, v}^{\star} r^{p}<t_{+}-\theta_{+, u} r^{p} .
$$

Thus by continuity there is a largest $t_{+}^{\star}$ such that

$$
t_{+}^{\star}-\theta_{+, v}^{\star} r^{p}=t_{+}-\theta_{+, u} r^{p} \text {. }
$$


With $t_{+}^{\star}$ at hand we now apply Lemma 9.1 (with the same $\delta, \widetilde{\delta}$ as in the proof of Theorem 9.3) combining it with (9.4) to get

$$
\frac{1}{c_{5}} \frac{d(x, \partial \Omega)}{r} v\left(A_{-}\right) \leq v(x, t) \leq c_{5} \frac{d(x, \partial \Omega)}{r} v\left(A_{+}^{\star}\right)
$$

for $x \in B_{r}\left(x_{0}\right) \cap \Omega$ and $t_{0}+\left(2 c_{4} \theta_{-}-\left(\theta_{+, u}+\theta_{+, v}^{\star}\right)\right) r^{p}<t<t_{0}+\left(2 c_{4} \theta_{-}-\theta_{+, u}\right) r^{p}$. Combining (9.3) and (9.5) we have completed the proof.

9.3. Boundary measures in $C^{1,1}$-domains. We conclude the section by describing the fine properties of the boundary measure defined in (1.6). The first theorem tells that the induced measure is mutually absolutely continuous with respect to the surface measure of $S_{T}$.

Theorem 9.6. Under the hypothesis of Theorem 9.3,

$$
0<\liminf _{\varrho \rightarrow 0} \frac{\mu_{u}\left(Q_{\varrho}(x, t)\right)}{\varrho^{n+1}} \leq \limsup _{\varrho \rightarrow 0} \frac{\mu_{u}\left(Q_{\varrho}(x, t)\right)}{\varrho^{n+1}}<+\infty
$$

where $Q_{\varrho}(x, t):=B_{\varrho}(x) \times\left(t-\varrho^{2}, t\right)$, whenever $(x, t) \in V$,

$$
V:=\left(\partial \Omega \cap B_{r}\left(x_{0}\right)\right) \times\left(t_{0}+\left(2 c_{4} \theta_{-}-2 \theta_{+}\right) r^{p}, t_{0}+\left(2 c_{4} \theta_{-}-\theta_{+}\right) r^{p}\right) .
$$

In particular, $\mu_{u}$ is mutually absolutely continuous with respect to the surface measure of $S_{T}$ on $V$.

Proof. By Theorem 9.3 we have that

$$
\lambda_{-} d(x, \partial \Omega) \leq u(x, t) \leq \lambda_{+} d(x, \partial \Omega), \quad \lambda_{ \pm}:=c_{5}^{ \pm 1} \frac{u\left(A_{r}^{ \pm}\right)}{r}
$$

whenever $(x, t) \in Q$ with

$$
Q:=\left(\Omega \cap B_{r}\left(x_{0}\right)\right) \times\left(t_{0}+\left(2 c_{4} \theta_{-}-2 \theta_{+}\right) r^{p}, t_{0}+\left(2 c_{4} \theta_{-}-\theta_{+}\right) r^{p}\right)
$$

and $\theta_{ \pm}$are as in Theorem 9.3. We now pick a point $(y, s) \in S_{T} \cap \partial_{p} Q$. Choose $\varrho$ small enough so that $U_{\varrho}(y, s) \cap(\Omega \times \mathbb{R})$ is contained in $Q$, where

$$
U_{\varrho}(y, s):=B_{\varrho}(y) \times\left(s-\widetilde{\tau} \varrho^{2}, s+\widetilde{\tau} \varrho^{2}\right),
$$

$\widetilde{\tau}:=\lambda_{-}^{2-p} \max \left\{8 C_{4} C_{5}^{2-p}, 2\left(\tau_{0}+\tau_{1}\right)\right\}$ and $C_{4}, C_{5}$ and $\tau_{0}, \tau_{1}$ are as in Theorems 5.2 and 5.3. After a simple covering argument using (9.6), and Theorems 5.2 and 5.3, we find a constant $C \equiv C\left(p, n, M, \lambda_{ \pm}\right)$such that

$$
\frac{1}{C} \leq \frac{\mu_{u}\left(U_{\varrho / 2}(y, s)\right)}{\varrho^{n+1}} \leq C .
$$

Taking a possibly larger $C$, and a smaller $\varrho$, this actually implies that

$$
\frac{1}{C} \leq \frac{\mu_{u}\left(Q_{\varrho}(y, s)\right)}{\varrho^{n+1}} \leq C,
$$

uniformly for small enough $\varrho$. This proves the statement.

Remark 9.7. Note that in the same region $V$ as in Theorem 9.6 we have that the measure is doubling. Moreover note that Lemma 9.2 implies a Hopf-type result on this boundary cylinder $V$, thus together with the fact that solutions are $C^{1, \alpha}$ up to the boundary we get that the logarithm of the normal derivative on the boundary is Hölder continuous. Now arguing as in [4, (1.7)-(1.10)] we get, for $\left(x_{0}, t_{0}\right) \in V$ given, and $\epsilon \in(0,1)$, that

$$
\lim _{\varrho \rightarrow 0} \frac{\mu_{u}\left(Q_{\epsilon \varrho}\left(x_{0}, t_{0}\right)\right)}{\mu_{u}\left(Q_{\varrho}\left(x_{0}, t_{0}\right)\right)}=\epsilon^{n+1} .
$$

In particular, the measure $\mu_{u}$ is asymptotically optimal doubling. 


\section{REFERENCES}

[1] H. Aikawa, T. Kilpeläinen, N. Shanmugalingam and X. Zhong, Boundary Harnack principle for p-harmonic functions in smooth Euclidean domains, Pot. Anal. 26(3) (2007), 281-301.

[2] B. Avelin, On time dependent domains for the degenerate $p$-parabolic equation: Carleson estimate and Hölder continuity, to appear in Math. Ann. (2015), doi:10.1007/s00208-015-1226-8.

[3] B. Avelin, U. Gianazza and S. Salsa, Boundary estimates for certain degenerate and singular parabolic equations, to appear in J. Eur. Math. Soc. (2015), arXiv:1406.1039.

[4] B. Avelin., N.L.P. Lundström and K. Nyström, Optimal doubling, Reifenberg flatness and operators of p-Laplace type, Nonlinear Anal. 74(17) (2011), 5943-5955.

[5] B. Avelin and K. Nyström, Wolff-potential estimates and doubling of subelliptic $p$-harmonic measures. Nonlinear Anal. 85 (2013), 145-159.

[6] M.F. Bidaut-Véron, Self-similar solutions of the $p$-Laplace heat equation: the case when $p>2$. Proc. Roy. Soc. Edinburgh Sect. A 139(1) (2009), 1-43.

[7] A. Björn, J. Björn, U. Gianazza and M. Parviainen, Boundary regularity for degenerate and singular parabolic equations, Calc. Var. Partial Differential Equations 52(3) (2015), $797-827$.

[8] M. Bonforte and J.L. Vázquez, A priori estimates for fractional nonlinear degenerate diffusion equations on bounded domains, Arch. Ration. Mech. Anal. 218(1) (2015), 317-362.

[9] E. DiBenedetto, Degenerate parabolic equations, Springer Verlag, Series Universitext, New York, (1993).

[10] E. DiBenedetto, U. Gianazza and V. Vespri, Harnack estimates for quasi-linear degenerate parabolic differential equation, Acta Math., 200 (2008), 181-209.

[11] E. DiBenedetto, U. Gianazza and V. Vespri, Harnack's inequality for degenerate and singular parabolic equations, Springer Monographs in Mathematics, Springer Verlag, New York (2012).

[12] E. DiBenedetto, Y.C. Kwong and V. Vespri, Local space analyticity of solutions of certain singular parabolic equations, Indiana Univ. Math. J. 40(2), (1991), 741-765.

[13] E.B. Fabes, N. Garofalo and S. Salsa, Comparison theorems for temperatures in noncylindrical domains. (Italian summary), Atti Accad. Naz. Lincei Rend. Cl. Sci. Fis. Mat. Natur. $\mathbf{7 7}(8)$ (1985), 1-12.

[14] E.B. Fabes, N. Garofalo and S. Salsa, A backward Harnack inequality and Fatou theorem for non-negative solutions of parabolic equations., Illinois J. Math. 30(4) (1986), 536-565.

[15] E.B. Fabes and M.V. Safonov, Behavior near the boundary of positive solutions of second order parabolic equations, Proceedings of the conference dedicated to Professor Miguel de Guzmán (El Escorial, 1996). J. Fourier Anal. Appl. 3, Special Issue, (1997), 871-882.

[16] E.B. Fabes, M.V. Safonov and Y. Yuan, Behavior near the boundary of positive solutions of second order parabolic equations. II, Trans. Amer. Math. Soc. 351(12) (1999), 4947-4961.

[17] N. Garofalo, Second order parabolic equations in nonvariational forms: boundary Harnack principle and comparison theorems for nonnegative solutions, Ann. Mat. Pura Appl. (4) 138 (1984), 267-296.

[18] D. Jerison and C. Kenig, Boundary behavior of harmonic functions in non-tangentially accessible domains, Adv. Math. 46 (1982), 80-147.

[19] J. Kemper, Temperatures in several variables, kernel functions, representations and parabolic boundary values, Trans. Amer. Math. Soc. 167 (1972), 243-262.

[20] T. Kilpeläinen and P. Lindqvist, On the Dirichlet boundary value problem for a degenerate parabolic equation., SIAM J. Math. Anal. 27(3) (1996), 661-683.

[21] T. Kilpeläinen and X. Zhong, Growth of entire $\mathcal{A}$-subharmonic functions., Ann. Acad. Sci. Fenn. Math. 28(1) (2003), 181-192.

[22] T. Kuusi, Harnack estimates for weak supersolutions to nonlinear degenerate parabolic equations, Ann. Sc. Norm. Super. Pisa Cl. Sci. (5) 7(4) (2008), 673-716

[23] T. Kuusi, P. Lindqvist and M. Parviainen, Shadows of Infinities, arXiv: 1406.6309.

[24] T. Kuusi, G. Mingione and K. Nyström, A boundary Harnack inequality for singular equations of p-parabolic type. Proc. Amer. Math. Soc. 142(8) (2014), 2705-2719.

[25] T. Kuusi and M. Parviainen: Existence of solutions to degenerate Cauchy problems, Manuscripta Math. 128 (2009), 213-249.

[26] J. Lewis and K. Nyström, Boundary behavior for $p$-harmonic functions in Lipschitz and starlike Lipschitz ring domains. Ann. Sci. École Norm. Sup. (4) 40(5) (2007), 765-813.

[27] J. Lewis and K. Nyström, Boundary behavior and the Martin boundary problem for $p$ harmonic functions in Lipschitz domains. Ann. of Math. (2) 172(3) (2010), 1907-1948.

[28] G.M. Lieberman, Boundary and initial regularity for solutions of degenerate parabolic equations, Nonlinear Anal. 20(5) (1993), 551-569. 
[29] K. Nyström, The Dirichlet problem for second order parabolic operators, Indiana Univ. Math. J. 46 (1997), 183-245.

[30] K. Nyström, H. Persson and O. Sande, Boundary estimates for non-negative solutions to non-linear parabolic equations, Calc. Var. Partial Differential Equations 54(1) (2015), 847879 .

[31] S. Salsa, Some properties of nonnegative solutions of parabolic differential operators, Ann. Mat. Pura Appl. 128(4) (1981), 193-206.

[32] D. Stan and J.L. Vázquez, Asymptotic behavior of the doubly non-linear diffusion equation $u_{t}=\Delta_{p} u^{m}$ on bounded domains, Nonlinear Anal. 77 (2013), 1-32.

Benny Avelin, Department of Mathematics and Systems Analysis, Aalto University School of Science, FI-00076 Aalto, Finland

Benny Avelin, Department of Mathematics, Uppsala University, S-751 06 Uppsala, SWEDEN

E-mail address: benny.avelin@math.uu.se

Tuomo Kuusi, Department of Mathematics and Systems Analysis, Aalto University School of Science, FI-00076 Aalto, Finland

E-mail address: tuomo.kuusi@aalto.fi

Kaj Nyström, Department of Mathematics, Uppsala University, S-751 06 Uppsala, SWEDEN

E-mail address: kaj.nystrom@math.uu.se 